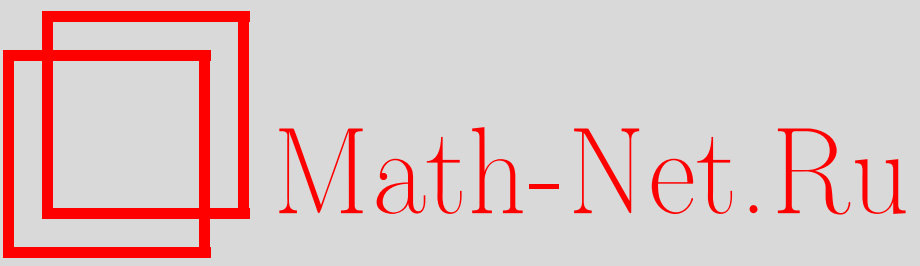

С. А. Духновский, О скорости стабилизации решений задачи Коши для системы уравнений Годунова-Султангазина с периодическими начальными данными, Итоги науки и техн. Сер. Соврем. мат. и ее прил. Темат. обз., 2019, том 165, 88-113

DOI: https://doi.org/10.36535/0233-6723-2019-165-88-113

Использование Общероссийского математического портала Math-Net.Ru подразумевает, что вы прочитали и согласны с пользовательским соглашением

http: //www. mathnet.ru/rus/agreement

Параметры загрузки:

IP : 54.237 .206 .68

26 апреля 2023 г., 15:40:24 


\title{
О СКОРОСТИ СТАБИЛИЗАЦИИ РЕШЕНИЙ ЗАДАЧИ КОШИ ДЛЯ СИСТЕМЫ УРАВНЕНИЙ ГОДУНОВА-СУЛТАНГАЗИНА С ПЕРИОДИЧЕСКИМИ НАЧАЛЬНЫМИ ДАННЫМИ
}

\author{
(c) 2019 г. \\ с. А. ДУХновСКИй
}

\begin{abstract}
АннотАция. Исследуется одномерная система уравнений для дискретной модели газа (система уравнений Годунова-Султангазина). Система Годунова-Султангазина является кинетическим уравнением Больцмана модельного одномерного газа, состоящего из трех групп частиц. Для этой модели сохраняются импульс, а энергия нет. Доказывается существование единственного глобального решения задачи Коши для возмущения состояния равновесия с периодическими начальными данными. Впервые устанавливается скорость стабилизации к состоянию равновесия (экспоненциальная стабилизация).
\end{abstract}

Ключевые слова: система Годунова-Султангазина, теорема существования, слабое решение, число Кнудсена.

\section{ON THE RATE OF STABILIZATION \\ OF SOLUTIONS TO THE CAUCHY PROBLEM \\ FOR THE GODUNOV-SULTANGAZIN SYSTEM \\ WITH PERIODIC INITIAL DATA}

\author{
(c) 2019 S. A. DUKHNOVSKY
}

\begin{abstract}
In this paper, we examine a one-dimensional system of equations for a discrete gas model (the Godunov-Sultangazin system). The Godunov-Sultangazin system is the Boltzmann kinetic equation for a model one-dimensional gas consisting of three groups of particles. In this model, the momentum is preserved whereas the energy is not. We prove the existence of a unique global solution to the Cauchy problem for a perturbation of the equilibrium state with periodic initial data. For the first time, we find the rate of stabilization to the equilibrium state (exponential stabilization).
\end{abstract}

Keywords and phrases: Godunov-Sultangazin system, existence theorem, weak solution, Knudsen number.

AMS Subject Classification: 35L45, 35L60, 35Q20

1. Введение. В настоящей работе мы продолжаем изучение дискретной кинетической модели Годунова-Султангазина (см. $[4,12])$, описывающей одноатомный разреженный газ, состоящий из трех групп частиц. Исследование данной системы началось в 1970-х гг. в работах С. К. Годунова и У. М. Султангазина (см. [4]. При этом доказательство глобальной теоремы существования решения в пространстве Соболева не приводилось. В более поздних работах обзор кинетических уравнений рассматривался В. В. Веденяпиным [3], О. А. Васильевой [1], Е. В. Радкевичем [7, 8], О. В. Ильиным [6] и многими другими авторами. 
Рассмотрим систему Годунова-Султангазина

$$
\begin{array}{ll}
\partial_{t} u+\partial_{x} u=\frac{1}{\varepsilon}\left(v^{2}-u w\right), & t>0, \quad x \in \mathbb{R}, \\
\partial_{t} v=-\frac{2}{\varepsilon}\left(v^{2}-u w\right), & 0<\varepsilon<1, \\
\partial_{t} w-\partial_{x} w=\frac{1}{\varepsilon}\left(v^{2}-u w\right), & \\
\left.u\right|_{t=0}=u^{0},\left.\quad v\right|_{t=0}=v^{0},\left.\quad w\right|_{t=0}=w^{0} .
\end{array}
$$

Здесь $u=u(x, t), v=v(x, t), w=w(x, t)$ - плотности групп частиц со скоростями $c=1,0,-1$, $\varepsilon$ - параметр Кнудсена из кинетической теории газов, $u^{0}(x)=u^{0}(x+2 \pi), v^{0}(x)=v^{0}(x+2 \pi)$, $w^{0}(x)=w^{0}(x+2 \pi)$ - периодические начальные данные. Приведенное в данной статье доказательство глобальной разрешимости задачи Коши для одномерной системы уравнений (1) позволяет исследовать природу локального равновесия и установить скорость стабилизации к состоянию равновесия решений задачи Коши с ограниченной энергией и периодическими начальными данными.

2. Фурье-решение. Мы исследуем задачу Коши (1) для малых возмущений состояния равновесия $v_{e}^{2}=w_{e} u_{e}, u_{e}, v_{e}, w_{e}>0$. Введем обозначение $\mathbb{Z}_{0}=\{k \in Z, k \neq 0\}$ и положим

$$
u=u_{e}+u_{e}^{1 / 2} \varepsilon^{2} \widehat{u}, \quad v=v_{e}+v_{e}^{1 / 2} \varepsilon^{2} \widehat{v}, \quad w=w_{e}+w_{e}^{1 / 2} \varepsilon^{2} \widehat{w},
$$

где

$$
\widehat{u}(t, x)=u_{0}(t)+\sum_{k \in \mathbb{Z}_{0}} u_{k}(t) e^{i k x}, \quad \widehat{v}(t, x)=v_{0}(t)+\sum_{k \in \mathbb{Z}_{0}} v_{k}(t) e^{i k x}, \quad \widehat{w}(t, x)=w_{0}(t)+\sum_{k \in \mathbb{Z}_{0}} w_{k}(t) e^{i k x},
$$

Тогда

$$
\begin{aligned}
& \partial_{t} \widehat{u}+\partial_{x} \widehat{u}-\frac{1}{\varepsilon} w_{e}^{1 / 2}\left(2 v_{e}^{1 / 2} \widehat{v}-u_{e}^{1 / 2} \widehat{w}-w_{e}^{1 / 2} \widehat{u}\right)=\varepsilon w_{e}^{1 / 2}\left(\widehat{v}^{2}-\widehat{u} \widehat{w}\right), \\
& \partial_{t} \widehat{v}+\frac{2}{\varepsilon} v_{e}^{1 / 2}\left(2 v_{e}^{1 / 2} \widehat{v}-u_{e}^{1 / 2} \widehat{w}-w_{e}^{1 / 2} \widehat{u}\right)=-2 \varepsilon v_{e}^{1 / 2}\left(\widehat{v}^{2}-\widehat{u} \widehat{w}\right), \\
& \partial_{t} \widehat{w}-\partial_{x} \widehat{w}-\frac{1}{\varepsilon} u_{e}^{1 / 2}\left(2 v_{e}^{1 / 2} \widehat{v}-u_{e}^{1 / 2} \widehat{w}-w_{e}^{1 / 2} \widehat{u}\right)=\varepsilon u_{e}^{1 / 2}\left(\widehat{v}^{2}-\widehat{u} \widehat{w}\right), \\
& \left.\widehat{u}\right|_{t=0}=\widehat{u}^{0},\left.\quad \widehat{v}\right|_{t=0}=\widehat{v}^{0},\left.\quad \widehat{w}\right|_{t=0}=\widehat{w}^{0} .
\end{aligned}
$$

Для периодических возмущений с нулевыми средними введем весовое пространство $W_{2, \gamma}^{1}\left(\mathbb{R}_{+} ; \mathcal{H}_{\sigma}\right)$ :

где

$$
\|\widehat{u}\|_{W_{2, \gamma}^{1}\left(\mathbb{R}_{+} ; \mathcal{H}_{\sigma}\right)}=\left\|\frac{d}{d t} \widehat{u}\right\|_{L_{2, \gamma}\left(\mathbb{R}_{+} ; \mathcal{H}_{\sigma}\right)}+\|\widehat{u}\|_{L_{2, \gamma}\left(\mathbb{R}_{+} ; \mathcal{H}_{\sigma}\right)}
$$

$$
\begin{gathered}
\|\widehat{u}\|_{L_{2, \gamma}\left(\mathbb{R}_{+} ; \mathcal{H}_{\sigma}\right)}^{2}= \\
\int_{0}^{\infty} e^{2 \gamma t}\left|u_{0}(t)\right|^{2} d t+\int_{0}^{\infty} e^{2 \gamma t} \sum_{k \in \mathbb{Z}_{0}}|k|^{2 \sigma}\left|u_{k}(t)\right|^{2} d t<\infty, \\
\left\|\left.\widehat{u}\right|_{t=0}\right\|_{\mathcal{H}_{\sigma}}^{2}=\left|u_{0}^{0}\right|^{2}+\sum_{k \in \mathbb{Z}_{0}}|k|^{2 \sigma}\left|u_{k}^{0}\right|^{2} .
\end{gathered}
$$

Сформулируем основной результат работы.

Теорема 1. Для любых $v_{e}^{2}=u_{e} w_{e}>0$ существуют такие не зависящие от $\varepsilon$ константы $\mu_{0}, q \in(0,1)$, что для мальх $0<\varepsilon<1$ и периодических начальных данных $\left(\widehat{u}^{0}, \widehat{v}^{0}, \widehat{w}^{0}\right)$ с нулевыми средними, для которых выполняется неравенство

$$
\left\|\widehat{u}^{0}\right\|_{\mathcal{H}_{\sigma}}+\left\|\left|\widehat{v}^{0}\left\|_{\mathcal{H}_{\sigma}}+\right\|\right| \widehat{w}^{0}\right\|_{\mathcal{H}_{\sigma}} \leqslant \varepsilon^{3 / 4} q
$$

для всех $\sigma>2$ существует единственное глобальное решение $(\widehat{u}, \widehat{v}, \widehat{w}) \in W_{2, \gamma}^{1}\left(\mathbb{R}_{+} ; \mathcal{H}_{\sigma}\right)$, где $\gamma=\varepsilon \mu_{0}>0$, задачи Коши (3).

Отсюда следует теорема об экспоненциальной устойчивости. 
Теорема 2. Пусть $\sigma>2$ и выполнены условия теоремы 1. Тогда положительное состояние равновесия $\left(u_{e}=\mathrm{const}>0, v_{e}=\mathrm{const}>0, w_{e}=\mathrm{const}>0, v_{e}^{2}=u_{e} w_{e}\right)$ является экспоненииально устойчивым:

$$
\begin{aligned}
\left\|u(x, t)-u_{e}\right\| \|_{\mathcal{H}_{\sigma}} & \leqslant c_{1}\left(\left\|\widehat{u}^{0}\right\|_{\mathcal{H}_{\sigma}}+\left\|\widehat{v}^{0}\right\|_{\mathcal{H}_{\sigma}}+\left\|\widehat{w}^{0}\right\|_{\mathcal{H}_{\sigma}}\right) e^{-2 \gamma_{1} t}, \\
\left\|v(x, t)-v_{e}\right\|_{\mathcal{H}_{\sigma}} & \leqslant c_{2}\left(\left\|\widehat{u}^{0}\right\|_{\mathcal{H}_{\sigma}}+\left\|\widehat{v}^{0}\right\|_{\mathcal{H}_{\sigma}}+\left\|\widehat{w}^{0}\right\|_{\mathcal{H}_{\sigma}}\right) e^{-2 \gamma_{1} t}, \\
\left\|w(x, t)-w_{e}\right\|_{\mathcal{H}_{\sigma}} & \leqslant c_{3}\left(\left\|\widehat{u}^{0}\right\|_{\mathcal{H}_{\sigma}}+\left\|\widehat{v}^{0}\right\|_{\mathcal{H}_{\sigma}}+\left\|\widehat{w}^{0}\right\|_{\mathcal{H}_{\sigma}}\right) e^{-2 \gamma_{1} t},
\end{aligned}
$$

где $c_{1}, c_{2}, c_{3}>0, \gamma_{1}>\gamma>0$.

Из данной теоремы 2 вытекает асимптотическая устойчивость. В [12] получена система для коэффициентов Фурье $\left\{u_{0}, v_{0}, w_{0}, u_{k}, v_{k}, w_{k}, k \in \mathbb{Z}_{0}\right\}$ :

$$
\begin{aligned}
& u_{k}=\left(u_{k}^{0}+\frac{1}{2} \frac{w_{e}^{1 / 2}}{v_{e}^{1 / 2}} v_{k}^{0}\right) e^{-i k t}-\frac{1}{2} \frac{w_{e}^{1 / 2}}{v_{e}^{1 / 2}} v_{k}+i k \frac{1}{2} \frac{w_{e}^{1 / 2}}{v_{e}^{1 / 2}} \int_{0}^{t} e^{i k(s-t)} v_{k} d s, \\
& w_{k}=\left(w_{k}^{0}+\frac{1}{2} \frac{u_{e}^{1 / 2}}{v_{e}^{1 / 2}} v_{k}^{0}\right) e^{i k t}-\frac{1}{2} \frac{u_{e}^{1 / 2}}{v_{e}^{1 / 2}} v_{k}-i k \frac{1}{2} \frac{u_{e}^{1 / 2}}{v_{e}^{1 / 2}} \int_{0}^{t} e^{-i k(s-t)} v_{k} d s, \\
& v_{k}=v_{k}^{0} e^{-L_{e} t / \varepsilon}+y_{k}, \quad k \in \mathbb{Z}_{0} .
\end{aligned}
$$

Потребуем, чтобы средние

$$
u_{0}^{0}=\frac{1}{2 \pi} \int_{0}^{2 \pi} \widehat{u}^{0}(x) d x=v_{0}^{0}=\frac{1}{2 \pi} \int_{0}^{2 \pi} \widehat{v}^{0}(x) d x=w_{0}^{0}=\frac{1}{2 \pi} \int_{0}^{2 \pi} \widehat{w}^{0}(x) d x=0 .
$$

Тогда

$$
u_{0}(t)=-\frac{1}{2} \frac{w_{e}^{1 / 2}}{v_{e}^{1 / 2}} v_{0}(t), \quad w_{0}(t)=-\frac{1}{2} \frac{u_{e}^{1 / 2}}{v_{e}^{1 / 2}} v_{0}(t) .
$$

В [12] доказано, что для $y_{k}$ имеет место бесконечная система обыкновенных дифференциальных уравнений

$$
\begin{gathered}
\frac{d}{d t} y_{k}+\frac{1}{\varepsilon} L_{e} y_{k}+\frac{1}{\varepsilon} i k \int_{0}^{t}\left(u_{e} e^{-i k(s-t)}-w_{e} e^{i k(s-t)}\right) y_{k} d s=2 \frac{1}{\varepsilon} v_{e}^{1 / 2} \mathcal{D}_{k}^{+} e^{i k t}+2 \frac{1}{\varepsilon} v_{e}^{1 / 2} \mathcal{D}_{k}^{-} e^{-i k t}+ \\
+e^{-L_{e} t / \varepsilon}\left(f_{k, L}(t)+f_{k, B}(t)\right)-\varepsilon v_{e}^{1 / 2} T_{k}^{\text {add }}(y)-\varepsilon v_{e}^{1 / 2}\left(\mathcal{L}_{k}(y)+B_{k}(y, y)\right), \\
\left.y_{k}\right|_{t=0}=0, \quad k \in \mathbb{Z}_{0} .
\end{gathered}
$$

Здесь $T_{k}^{\mathrm{add}}(y)$ - оператор возмущения базовой системы

$$
\begin{gathered}
\frac{d}{d t} y_{k}+\frac{1}{\varepsilon} L_{e} y_{k}+\frac{1}{\varepsilon} i k \int_{0}^{t}\left(u_{e} e^{-i k(s-t)}-w_{e} e^{i k(s-t)}\right) y_{k} d s=2 \frac{1}{\varepsilon} v_{e}^{1 / 2} \mathcal{D}_{k}^{+} e^{i k t}+2 \frac{1}{\varepsilon} v_{e}^{1 / 2} \mathcal{D}_{k}^{-} e^{-i k t}+ \\
+e^{-L_{e} t / \varepsilon}\left(f_{k, L}(t)+f_{k, B}(t)\right)-\varepsilon v_{e}^{1 / 2}\left(\mathcal{L}_{k}(y)+B_{k}(y, y)\right), \\
\left.y_{k}\right|_{t=0}=0, \quad k \in \mathbb{Z}_{0} .
\end{gathered}
$$

Выражения $\mathcal{D}_{k}^{+}, \mathcal{D}_{k}^{-}, f_{k, L}(t), f_{k, B}(t), \mathcal{L}_{k}(y), B_{k}(y, y)$ определены в [12]. Далее будет рассматриваться невозмущенная система (5). Системе (4) с оператором возмущения будет посвящена другая публикация. 
3. Конечная аппроксимация. Для построения аппроксимационного решения задачи Коши (3) в [12] введена конечная аппроксимация бесконечной системы (5):

$$
\begin{gathered}
\frac{d}{d t} y_{k}^{(m)}+\frac{1}{\varepsilon} L_{e} y_{k}^{(m)}+\frac{1}{\varepsilon} i k \int_{0}^{t}\left(u_{e} e^{-i k(s-t)}-w_{e} e^{i k(s-t)}\right) y_{k}^{(m)} d s=2 \frac{1}{\varepsilon} v_{e}^{1 / 2} \mathcal{D}_{k, m}^{+} e^{i k t}+ \\
+2 \frac{1}{\varepsilon} v_{e}^{1 / 2} \mathcal{D}_{k, m}^{-} e^{-i k t}+e^{-L_{e} t / \varepsilon}\left(f_{k, L}^{(m)}(t)+f_{k, B}^{(m)}(t)\right)-\varepsilon v_{e}^{1 / 2}\left(\mathcal{L}_{k}^{(m)}\left(y^{(m)}\right)+B_{k}^{(m)}\left(y^{(m)}, y^{(m)}\right)\right), \\
\left.y_{k}^{(m)}\right|_{t=0}=0, \quad|k|=1, \ldots, m .
\end{gathered}
$$

В правой части (6) появляются секулярные члены $\frac{2}{\varepsilon} v_{e}^{1 / 2} \mathcal{D}_{k, m}^{+} e^{i k t}, \frac{2}{\varepsilon} v_{e}^{1 / 2} \mathcal{D}_{k, m}^{-} e^{-i k t}$, не принадлежащие $L_{2, \gamma}\left(\mathbb{R}_{+}\right)$. Введем векторное пространство $Q^{(m)}=\left(Q_{k}^{(m)},|k| \leqslant m, k \neq 0\right) \in \mathcal{H}_{\sigma}^{(m)}$ с нормой

$$
\left\|\left.\left|Q^{(m)} \|_{\mathcal{H}_{\sigma}^{(m)}}^{2}=\sum_{\substack{k \in \mathbb{Z}_{0},|k| \leqslant m}}\right| k\right|^{2 \sigma}\left|Q_{k}^{(m)}\right|^{2} .\right.
$$

Решение системы (6) будем искать в виде

$$
\begin{aligned}
y_{k}^{(m)}= & Q_{k, m}^{+} T_{k}^{-1}\left(e^{i k t}\right)+Q_{k, m}^{-} T_{k}^{-1}\left(e^{-i k t}\right)+T_{k}^{-1}\left(z_{k}^{(m)}\right),\left.\quad z_{k}^{(m)}\right|_{t=0}=0, \quad k \in \mathbb{Z}_{0}, \\
& Q_{k, m}^{+}, Q_{k, m}^{-} \in \mathcal{H}_{\sigma}^{(m)}, \quad Z^{(m)}=\left\{z_{k}^{(m)},|k| \leqslant m\right\} \in L_{2, \gamma}\left(\mathbb{R}_{+} ; \mathcal{H}_{\sigma}^{(m)}\right) .
\end{aligned}
$$

В переменных $\left(z_{k}^{(m)}, Q_{k, m}^{+}, Q_{k, m}^{-}\right)$(см. [12]) система (6) при выполнении условий секулярности

$$
\begin{aligned}
& Q_{k, m}^{+}=\frac{2}{\varepsilon} v_{e}^{1 / 2} \mathcal{D}_{k, m}^{+}-\varepsilon v_{e}^{1 / 2} S^{+}\left(Q_{m}^{-}, Q_{m}^{+}\right), \\
& Q_{k, m}^{-}=\frac{2}{\varepsilon} v_{e}^{1 / 2} \mathcal{D}_{k, m}^{-}-\varepsilon v_{e}^{1 / 2} S^{-}\left(Q_{m}^{-}, Q_{m}^{+}\right), \quad|k|=1, \ldots, m,
\end{aligned}
$$

запишется в виде:

$$
\begin{aligned}
z_{k}^{(m)}=e^{-L_{e} t / \varepsilon}\left(F_{k, L}^{(m)}(t)+F_{k, B}^{(m)}(t)\right) & -\varepsilon v_{e}^{1 / 2}\left(H_{k, L}^{(m)}(t)+H_{k, B}^{(m)}(t)\right)- \\
& -\varepsilon v_{e}^{1 / 2}\left(\mathcal{U}_{k}^{(m)}\left(T^{-1}\left(Z^{(m)}\right)\right)+B_{k}^{(m)}\left(T^{-1}\left(Z^{(m)}\right), T^{-1}\left(Z^{(m)}\right)\right)\right),
\end{aligned}
$$

где

$$
\begin{aligned}
& 2 v_{e}^{1 / 2} \frac{1}{\varepsilon} \mathcal{D}_{k, m}^{-}=2 v_{e}^{1 / 2} w_{e}^{1 / 2} \frac{1}{\varepsilon}\left(u_{k}^{0}-\frac{1}{2} \frac{w_{e}^{1 / 2}}{v_{e}^{1 / 2}} \frac{L_{e} / \varepsilon}{\left(i k-L_{e} / \varepsilon\right)} v_{k}^{0}\right)+ \\
& +\varepsilon v_{e}^{1 / 2} \sum_{\substack{k_{1}+k_{2}=k,\left|k_{1}\right|,\left|k_{2}\right|=1, \ldots, m}} \overline{\left(w_{k_{2}}^{0}+\frac{1}{2} \frac{u_{e}^{1 / 2}}{v_{e}^{1 / 2}} \frac{L_{e} / \varepsilon}{\left(i k_{2}+L_{e} / \varepsilon\right)} v_{k_{2}}^{0}\right)}\left(u_{k_{1}}^{0}-\frac{1}{2} \frac{L_{e} / \varepsilon}{\left(i k_{1}-L_{e} / \varepsilon\right)} \frac{w_{e}^{1 / 2}}{v_{e}^{1 / 2}} v_{k_{1}}^{0}\right), \\
& 2 v_{e}^{1 / 2} \frac{1}{\varepsilon} \mathcal{D}_{k, m}^{+}=2 v_{e}^{1 / 2} u_{e}^{1 / 2} \frac{1}{\varepsilon}\left(w_{k}^{0}+\frac{1}{2} \frac{L_{e} / \varepsilon}{\left(i k+L_{e} / \varepsilon\right)} \frac{u_{e}^{1 / 2}}{v_{e}^{1 / 2}} v_{k}^{0}\right)+ \\
& +\varepsilon v_{e}^{1 / 2} \sum_{\substack{k_{1}+k_{2}=k,\left|k_{1}\right|,\left|k_{2}\right|=1, \ldots, m}}\left(w_{k_{2}}^{0}+\frac{1}{2} \frac{L_{e} / \varepsilon}{\left(i k_{2}+L_{e} / \varepsilon\right)} \frac{u_{e}^{1 / 2}}{v_{e}^{1 / 2}} v_{k}^{0}\right) \overline{\left(u_{k_{1}}^{0}-\frac{1}{2} \frac{w_{e}^{1 / 2}}{v_{e}^{1 / 2}} \frac{L_{e} / \varepsilon}{\left(i k_{1}-L_{e} / \varepsilon\right)} v_{k_{1}}^{0}\right)}, \\
& S^{+}\left(Q_{m}^{-}, Q_{m}^{+}\right)= \\
& =\frac{1}{2} \sum_{\substack{k_{1}+k_{2}=k,\left|k_{1}\right|,\left|k_{2}\right|=1, \ldots, m}}\left\{\left(\overline{u_{k_{1}}^{0}}+\frac{1}{2} \frac{w_{e}^{1 / 2}}{v_{e}^{1 / 2}} \overline{k_{1}}\right) \frac{\varepsilon}{u_{e}} \frac{u_{e}^{1 / 2}}{v_{e}^{1 / 2}} Q_{k_{2}, m}^{+}+\left(w_{k_{2}}^{0}+\frac{1}{2} \frac{u_{e}^{1 / 2}}{v_{e}^{1 / 2}} v_{k_{2}}^{0}\right) \frac{\varepsilon}{w_{e}} \frac{w_{e}^{1 / 2}}{v_{e}^{1 / 2}} \overline{Q_{k_{1}, m}^{-}}\right)-
\end{aligned}
$$




$$
\left.-\frac{1}{2}\left(\frac{i k_{2}}{\left(i k_{2}+L_{e} / \varepsilon\right)} v_{k_{2}}^{0} \frac{\varepsilon}{w_{e}} \overline{Q_{k_{1}, m}^{-}}+\frac{\varepsilon}{u_{e}} Q_{k_{2}, m}^{+} \frac{i k_{1}}{\left(i k_{1}+L_{e} / \varepsilon\right)} \overline{v_{k_{1}}^{0}}\right)-\frac{1}{2} \frac{\varepsilon}{w_{e}} \frac{\varepsilon}{u_{e}} Q_{k_{2}, m}^{+} \overline{Q_{k_{1}, m}^{-}}\right\}
$$

$$
\begin{gathered}
S^{-}\left(Q_{m}^{-}, Q_{m}^{+}\right)= \\
\begin{array}{r}
\frac{1}{2} \sum_{\substack{k_{1}+k_{2}=k,\left|k_{1}\right|,\left|k_{2}\right|=1, \ldots, m}}\left\{\left(\overline{w_{k_{2}}^{0}}+\frac{1}{2} \frac{u_{e}^{1 / 2}}{v_{e}^{1 / 2}} \overline{v_{k_{2}}^{0}}\right) \frac{w_{e}^{1 / 2}}{v_{e}^{1 / 2}} \frac{\varepsilon}{w_{e}} Q_{k_{1}, m}^{-}+\left(u_{k_{1}}^{0}+\frac{1}{2} \frac{w_{e}^{1 / 2}}{v_{e}^{1 / 2}} v_{k_{1}}^{0}\right) \frac{\varepsilon}{u_{e}} \frac{\left.w_{e}^{1 / 2} \overline{v_{e}} \overline{Q_{k_{2}, m}^{+}}\right)+}{-} \frac{1}{2}\left(\frac{i k_{1}}{\left(i k_{1}-L_{e} / \varepsilon\right)} v_{k_{1}}^{0} \frac{\varepsilon}{u_{e}} \overline{Q_{k_{2}, m}^{+}}+\frac{\varepsilon}{w_{e}} Q_{k_{1}, m}^{-} \frac{i k_{2}}{\left(i k_{2}-L_{e} / \varepsilon\right)} \overline{v_{k_{2}}^{0}}\right)-\frac{1}{2} \frac{\varepsilon}{w_{e}} \frac{\varepsilon}{u_{e}} Q_{k_{1}, m}^{-} \overline{Q_{k_{2}, m}^{+}}\right\}, \\
\quad+B_{k}^{(m)}\left(T^{-1}\left(D^{(m)}\right), T^{-1}\left(Z^{(m)}\right)\right)+B_{k}^{(m)}\left(T^{-1}\left(Z^{(m)}\right), T^{-1}\left(D^{(m)}\right)\right), \\
\mathcal{U}_{k}^{(m)}\left(T^{-1}\left(Z^{(m)}\right)\right)=L_{k}^{(m)}\left(T^{-1}\left(Z^{(m)}\right)\right)+ \\
D^{(m)}=\left(D_{k}^{(m)}, k \in \mathbb{Z}_{0},|k| \leqslant m\right), \quad D_{k}^{(m)}=Q_{k, m}^{-} e^{-i k t}+Q_{k, m}^{+} e^{i k t}, \\
F_{k, B}^{(m)}(t)=f_{k, B}^{(m)}(t)+F_{k}^{B, 2}(t)+F_{k}^{B, 1}(t), \quad F_{k, L}^{(m)}(t)=f_{k, L}^{(m)}(t) .
\end{array}
\end{gathered}
$$

Уравнение для нулевой моды в гильбертовом пространстве $L_{2, \gamma}\left(\mathbb{R}_{+}\right)$, при выполнении условия секулярности

$$
\mathcal{D}_{0}\left(Q_{m}^{+}, Q_{m}^{-}\right)=D_{0}-D_{0}^{(1)}=0,
$$

имеет следующий вид:

$$
\begin{aligned}
z_{0}^{(m)} & =-\frac{3}{2} \varepsilon v_{e}^{1 / 2} \int_{0}^{t} e^{L_{e}(s-t) / \varepsilon}\left[z_{0}^{(m)} \overline{z_{0}^{(m)}}+\right. \\
& \left.+\frac{2}{3}\left(\mathcal{L}_{0}^{(1)}\left(T^{-1}\left(Z^{(m)}\right)\right)+B_{0}^{(m)}\left(T^{-1}\left(Z^{(m)}\right), T^{-1}\left(Z^{(m)}\right)\right)+f_{0}(s)^{(m)} e^{-L_{e} s / \varepsilon}+h_{0}^{(m)}(s)\right)\right] d s
\end{aligned}
$$

где

$$
\begin{aligned}
& D_{0}^{(1)}=\frac{1}{2} \sum_{\substack{k_{1}+k_{2}=0, k_{1}, k_{2} \neq 0}}\left\{\left(u_{k_{1}}^{0}-\frac{1}{2} \frac{w_{e}^{1 / 2}}{v_{e}^{1 / 2}} \frac{L_{e} / \varepsilon}{\left(i k_{1}-L_{e} / \varepsilon\right)} v_{k_{1}}^{0}\right) \frac{\varepsilon}{u_{e}} \frac{u_{e}^{1 / 2}}{v_{e}^{1 / 2}} \overline{Q_{k_{2}, m}^{+}}+\right. \\
& +\frac{u_{e}^{1 / 2}}{v_{e}^{1 / 2}} \frac{\varepsilon}{u_{e}} Q_{k_{2}, m}^{+} \overline{\left(u_{k_{1}}^{0}-\frac{1}{2} \frac{w_{e}^{1 / 2}}{v_{e}^{1 / 2}} \frac{L_{e} / \varepsilon}{\left(i k_{1}-L_{e} / \varepsilon\right)} v_{k_{1}}^{0}\right)}+\frac{w_{e}^{1 / 2}}{v_{e}^{1 / 2}} \frac{\varepsilon}{w_{e}} Q_{k_{1}, m}^{-} \overline{\left(w_{k_{2}}^{0}+\frac{1}{2} \frac{u_{e}^{1 / 2}}{v_{e}^{1 / 2}} \frac{L_{e} / \varepsilon}{\left(i k_{2}+L_{e} / \varepsilon\right)} v_{k_{2}}^{0}\right)}+
\end{aligned}
$$

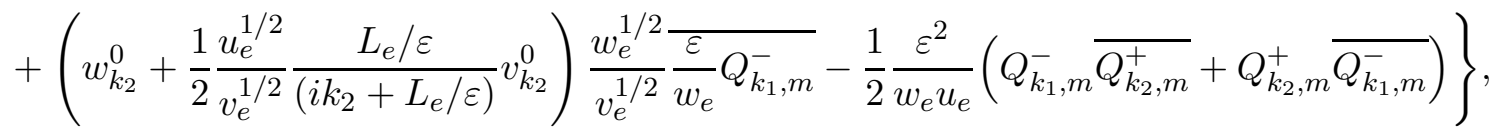

$$
\begin{aligned}
& \mathcal{L}_{0}^{(1)}\left(T^{-1}\left(Z^{(m)}\right)\right)=B_{0}^{(m)}\left(T^{-1}\left(D^{(m)}\right), T^{-1}\left(Z^{(m)}\right)\right)+ \\
& +B_{0}^{(m)}\left(T^{-1}\left(Z^{(m)}\right), T^{-1}\left(D^{(m)}\right)\right)+\mathcal{L}_{0}\left(T^{-1}\left(Z^{(m)}\right)\right) .
\end{aligned}
$$


4. Проверка условий секулярности. Проверим уравнения секулярности для нулевой и ненулевой моды. Решение уравнений секулярности (см. [12]) имеет вид

$$
\begin{aligned}
Q_{k, m}^{+} & =2 v_{e}^{1 / 2} u_{e}^{1 / 2} \frac{1}{\varepsilon}\left(w_{k}^{0}+\frac{1}{2} \frac{\frac{1}{\varepsilon} L_{e}}{\left(i k+L_{e} / \varepsilon\right)} \frac{u_{e}^{1 / 2}}{v_{e}^{1 / 2}} v_{k}^{0}\right), \\
Q_{k, m}^{-} & =2 v_{e}^{1 / 2} w_{e}^{1 / 2} \frac{1}{\varepsilon}\left(u_{k}^{0}-\frac{1}{2} \frac{w_{e}^{1 / 2}}{v_{e}^{1 / 2}} \frac{L_{e} / \varepsilon}{\left(i k-L_{e} / \varepsilon\right)} v_{k}^{0}\right) .
\end{aligned}
$$

Имеем для $S^{+}\left(Q_{m}^{-}, Q_{m}^{+}\right)$:

$$
\begin{aligned}
& S^{+}\left(Q_{m}^{-}, Q_{m}^{+}\right)= \\
& =\frac{1}{2} \sum_{\substack{k_{1}+k_{2}=k,\left|k_{1}\right|,\left|k_{2}\right|=1, \ldots, m}}\left\{\left(\overline{u_{k_{1}}^{0}}+\frac{1}{2} \frac{w_{e}^{1 / 2}}{v_{e}^{1 / 2}} \overline{v_{k_{1}}^{0}}\right) \frac{\varepsilon}{u_{e}} \frac{u_{e}^{1 / 2}}{v_{e}^{1 / 2}} Q_{k_{2}, m}^{+}+\left(w_{k_{2}}^{0}+\frac{1}{2} \frac{u_{e}^{1 / 2}}{v_{e}^{1 / 2}} v_{k_{2}}^{0}\right) \frac{\varepsilon}{w_{e}} \frac{w_{e}^{1 / 2} \overline{v_{e}} \overline{Q_{k_{1}, m}^{-}}}{v^{1 / 2}}\right)- \\
& \left.-\frac{1}{2}\left(\frac{i k_{2}}{\left(i k_{2}+L_{e} / \varepsilon\right)} v_{k_{2}}^{0} \frac{\varepsilon}{w_{e}} \overline{Q_{k_{1}, m}^{-}}+\frac{\varepsilon}{u_{e}} Q_{k_{2}, m}^{+} \frac{i k_{1}}{\left(i k_{1}+L_{e} / \varepsilon\right)} \overline{v_{k_{1}}^{0}}\right)-\frac{1}{2} \frac{\varepsilon}{w_{e}} \frac{\varepsilon}{u_{e}} Q_{k_{2}, m}^{+} \overline{Q_{k_{1}, m}^{-}}\right\}= \\
& =\frac{1}{2} \sum_{\substack{k_{1}+k_{2}=k,\left|k_{1}\right|,\left|k_{2}\right|=1, \ldots, m}}\left\{\left(\overline{u_{k_{1}}^{0}}+\frac{1}{2} \frac{w_{e}^{1 / 2}}{v_{e}^{1 / 2}} \overline{v_{k_{1}}^{0}}\right) \frac{\varepsilon}{u_{e}} \frac{u_{e}^{1 / 2}}{v_{e}^{1 / 2}} 2 \frac{u_{e}^{1 / 2} v_{e}^{1 / 2}}{\varepsilon}\left(w_{k_{2}}^{0}+\frac{1}{2} \frac{u_{e}^{1 / 2}}{v_{e}^{1 / 2}} \frac{L_{e} / \varepsilon}{\left(i k_{2}+L_{e} / \varepsilon\right)} v_{k_{2}}^{0}\right)+\right. \\
& \left.+\left(w_{k_{2}}^{0}+\frac{1}{2} \frac{u_{e}^{1 / 2}}{v_{e}^{1 / 2}} v_{k_{2}}^{0}\right) \frac{\varepsilon}{w_{e}} \frac{w_{e}^{1 / 2}}{v_{e}^{1 / 2}} 2 \overline{\frac{w_{e}^{1 / 2} v_{e}^{1 / 2}}{\varepsilon}\left(u_{k_{1}}^{0}-\frac{1}{2} \frac{w_{e}^{1 / 2}}{v_{e}^{1 / 2}} \frac{L_{e} / \varepsilon}{i k_{1}-L_{e} / \varepsilon} v_{k_{1}}^{0}\right)}\right)- \\
& -\frac{1}{2}\left(\frac{i k_{2}}{\left(i k_{2}+L_{e} / \varepsilon\right)} v_{k_{2}}^{0} \frac{\varepsilon}{w_{e}} 2 \overline{\frac{w_{e}^{1 / 2} v_{e}^{1 / 2}}{\varepsilon}\left(u_{k_{1}}^{0}-\frac{1}{2} \frac{w_{e}^{1 / 2}}{v_{e}^{1 / 2}} \frac{L_{e} / \varepsilon}{i k_{1}-L_{e} / \varepsilon} v_{k_{1}}^{0}\right)}+\right. \\
& \left.+\frac{\varepsilon}{u_{e}} 2 \frac{u_{e}^{1 / 2} v_{e}^{1 / 2}}{\varepsilon}\left(w_{k_{2}}^{0}+\frac{1}{2} \frac{u_{e}^{1 / 2}}{v_{e}^{1 / 2}} \frac{L_{e} / \varepsilon}{\left(i k_{2}+L_{e} / \varepsilon\right)} v_{k_{2}}^{0}\right) \frac{i k_{1}}{\left(i k_{1}+L_{e} / \varepsilon\right)} \overline{v_{k_{1}}^{0}}\right)- \\
& \left.-\frac{1}{2} \frac{\varepsilon}{w_{e}} \frac{\varepsilon}{u_{e}} 2 \frac{u_{e}^{1 / 2} v_{e}^{1 / 2}}{\varepsilon}\left(w_{k_{2}}^{0}+\frac{1}{2} \frac{u_{e}^{1 / 2}}{v_{e}^{1 / 2}} \frac{L_{e} / \varepsilon}{\left(i k_{2}+L_{e} / \varepsilon\right)} v_{k_{2}}^{0}\right) \overline{2 \frac{w_{e}^{1 / 2} v_{e}^{1 / 2}}{\varepsilon}\left(u_{k_{1}}^{0}-\frac{1}{2} \frac{w_{e}^{1 / 2}}{v_{e}^{1 / 2}} \frac{L_{e} / \varepsilon}{i k_{1}-L_{e} / \varepsilon} v_{k_{1}}^{0}\right)}\right\}=
\end{aligned}
$$

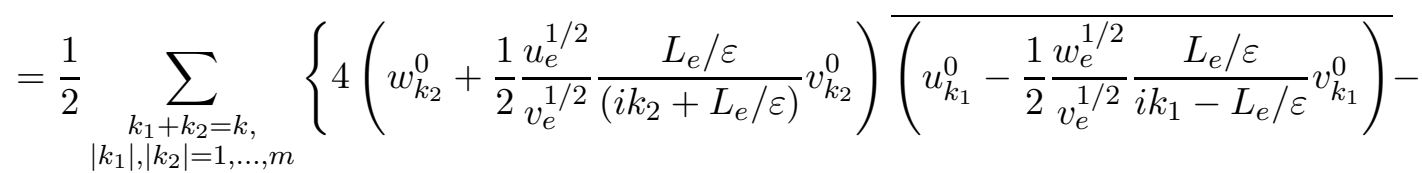

$$
\begin{aligned}
& \left.-2\left(w_{k_{2}}^{0}+\frac{1}{2} \frac{u_{e}^{1 / 2}}{v_{e}^{1 / 2}} \frac{L_{e} / \varepsilon}{\left(i k_{2}+L_{e} / \varepsilon\right)} v_{k_{2}}^{0}\right) \overline{\left(u_{k_{1}}^{0}-\frac{1}{2} \frac{w_{e}^{1 / 2}}{v_{e}^{1 / 2}} \frac{L_{e} / \varepsilon}{i k_{1}-L_{e} / \varepsilon} v_{k_{1}}^{0}\right)}\right\}= \\
& =\sum_{\substack{k_{1}+k_{2}=k,\left|k_{1}\right|,\left|k_{2}\right|=1, \ldots, m}}\left(w_{k_{2}}^{0}+\frac{1}{2} \frac{u_{e}^{1 / 2}}{v_{e}^{1 / 2}} \frac{L_{e} / \varepsilon}{\left(i k_{2}+L_{e} / \varepsilon\right)} v_{k_{2}}^{0}\right) \overline{\left(u_{k_{1}}^{0}-\frac{1}{2} \frac{w_{e}^{1 / 2}}{v_{e}^{1 / 2}} \frac{L_{e} / \varepsilon}{i k_{1}-L_{e} / \varepsilon} v_{k_{1}}^{0}\right)} .
\end{aligned}
$$

Тогда

$$
\begin{aligned}
& 2 v_{e}^{1 / 2} u_{e}^{1 / 2} \frac{1}{\varepsilon}\left(w_{k}^{0}+\frac{1}{2} \frac{L_{e} / \varepsilon}{\left(i k+L_{e} / \varepsilon\right)} \frac{u_{e}^{1 / 2}}{v_{e}^{1 / 2}} v_{k}^{0}\right)=2 v_{e}^{1 / 2} u_{e}^{1 / 2} \frac{1}{\varepsilon}\left(w_{k}^{0}+\frac{1}{2} \frac{L_{e} / \varepsilon}{\left(i k+L_{e} / \varepsilon\right)} \frac{u_{e}^{1 / 2}}{v_{e}^{1 / 2}} v_{k}^{0}\right)+ \\
&+\varepsilon v_{e}^{1 / 2} \sum_{\substack{k_{1}+k_{2}=k,\left|k_{1}\right|,\left|k_{2}\right|=1, \ldots, m}}\left(w_{k_{2}}^{0}+\frac{1}{2} \frac{L_{e} / \varepsilon}{\left(i k_{2}+L_{e} / \varepsilon\right)} \frac{u_{e}^{1 / 2}}{v_{e}^{1 / 2}} v_{k}^{0}\right)\left(u_{k_{1}}^{0}-\frac{1}{2} \frac{w_{e}^{1 / 2}}{v_{e}^{1 / 2}} \frac{L_{e} / \varepsilon}{\left(i k_{1}-L_{e} / \varepsilon\right)} v_{k_{1}}^{0}\right)-
\end{aligned}
$$




$$
-\varepsilon v_{e}^{1 / 2} \sum_{\substack{k_{1}+k_{2}=k,\left|k_{1}\right|,\left|k_{2}\right|=1, \ldots, m}}\left(w_{k_{2}}^{0}+\frac{1}{2} \frac{u_{e}^{1 / 2}}{v_{e}^{1 / 2}} \frac{L_{e} / \varepsilon}{\left(i k_{2}+L_{e} / \varepsilon\right)} v_{k_{2}}^{0}\right) \overline{\left(u_{k_{1}}^{0}-\frac{1}{2} \frac{w_{e}^{1 / 2}}{v_{e}^{1 / 2}} \frac{L_{e} / \varepsilon}{i k_{1}-L_{e} / \varepsilon} v_{k_{1}}^{0}\right)} .
$$

Отсюда получаем, что

$$
2 v_{e}^{1 / 2} u_{e}^{1 / 2} \frac{1}{\varepsilon}\left(w_{k}^{0}+\frac{1}{2} \frac{L_{e} / \varepsilon}{\left(i k+L_{e} / \varepsilon\right)} \frac{u_{e}^{1 / 2}}{v_{e}^{1 / 2}} v_{k}^{0}\right)-2 v_{e}^{1 / 2} u_{e}^{1 / 2} \frac{1}{\varepsilon}\left(w_{k}^{0}+\frac{1}{2} \frac{L_{e} / \varepsilon}{\left(i k+L_{e} / \varepsilon\right)} \frac{u_{e}^{1 / 2}}{v_{e}^{1 / 2}} v_{k}^{0}\right)=0
$$

что соответствует выполнению уравнения секулярности (7) невозмущенной задачи (6). Аналогично проводится проверка для (8).

Проверим выполнение условия секулярности $\mathcal{D}_{0}\left(Q_{m}^{+}, Q_{m}^{-}\right)=0$ для нулевой моды. А именно, подставим найденное в [12] решение $Q_{m}^{+}$и $Q_{m}^{-}$в $\mathcal{D}_{0}\left(Q_{m}^{+}, Q_{m}^{-}\right)$. Преобразуем $D_{0}^{(1)}$ :

$$
\begin{aligned}
& D_{0}^{(1)}\left(Q_{m}^{+}, Q_{m}^{-}\right)= \\
& =\frac{1}{2} \sum_{\substack{k_{1}+k_{2}=0, k_{1}, k_{2} \neq 0}}\left\{\left(u_{k_{1}}^{0}-\frac{1}{2} \frac{w_{e}^{1 / 2}}{v_{e}^{1 / 2}} \frac{L_{e} / \varepsilon}{\left(i k_{1}-L_{e} / \varepsilon\right)} v_{k_{1}}^{0}\right) \frac{\varepsilon}{u_{e}} \frac{u_{e}^{1 / 2}}{v_{e}^{1 / 2}} 2 \frac{u_{e}^{1 / 2} v_{e}^{1 / 2}}{\varepsilon} \overline{\left(w_{k_{2}}^{0}+\frac{1}{2} \frac{u_{e}^{1 / 2}}{v_{e}^{1 / 2}} \frac{L_{e} / \varepsilon}{\left(i k_{2}+L_{e} / \varepsilon\right)} v_{k_{2}}^{0}\right)}+\right. \\
& +\frac{u_{e}^{1 / 2}}{v_{e}^{1 / 2}} \frac{\varepsilon}{u_{e}} 2 \frac{u_{e}^{1 / 2} v_{e}^{1 / 2}}{\varepsilon}\left(w_{k_{2}}^{0}+\frac{1}{2} \frac{u_{e}^{1 / 2}}{v_{e}^{1 / 2}} \frac{L_{e} / \varepsilon}{\left(i k_{2}+L_{e} / \varepsilon\right)} v_{k_{2}}^{0}\right) \overline{\left(u_{k_{1}}^{0}-\frac{1}{2} \frac{w_{e}^{1 / 2}}{v_{e}^{1 / 2}} \frac{L_{e} / \varepsilon}{\left(i k_{1}-L_{e} / \varepsilon\right)} v_{k_{1}}^{0}\right)}+ \\
& +\frac{w_{e}^{1 / 2}}{v_{e}^{1 / 2}} \frac{\varepsilon}{w_{e}} 2 \frac{w_{e}^{1 / 2} v_{e}^{1 / 2}}{\varepsilon}\left(u_{k_{1}}^{0}-\frac{1}{2} \frac{w_{e}^{1 / 2}}{v_{e}^{1 / 2}} \frac{L_{e} / \varepsilon}{i k_{1}-L_{e} / \varepsilon} v_{k_{1}}^{0}\right) \overline{\left(w_{k_{2}}^{0}+\frac{1}{2} \frac{u_{e}^{1 / 2}}{v_{e}^{1 / 2}} \frac{L_{e} / \varepsilon}{\left(i k_{2}+L_{e} / \varepsilon\right)} v_{k_{2}}^{0}\right)}+ \\
& +\left(w_{k_{2}}^{0}+\frac{1}{2} \frac{u_{e}^{1 / 2}}{v_{e}^{1 / 2}} \frac{L_{e} / \varepsilon}{\left(i k_{2}+L_{e} / \varepsilon\right)} v_{k_{2}}^{0}\right) \frac{w_{e}^{1 / 2}}{v_{e}^{1 / 2}} \frac{\varepsilon}{w_{e}} 2 \frac{w_{e}^{1 / 2} v_{e}^{1 / 2}}{\varepsilon}\left(u_{k_{1}}^{0}-\frac{1}{2} \frac{w_{e}^{1 / 2}}{v_{e}^{1 / 2}} \frac{L_{e} / \varepsilon}{i k_{1}-L_{e} / \varepsilon} v_{k_{1}}^{0}\right)-
\end{aligned}
$$

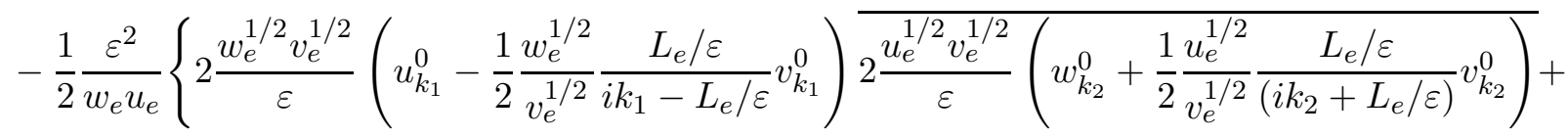

$$
\begin{aligned}
& \left.\left.+2 \frac{u_{e}^{1 / 2} v_{e}^{1 / 2}}{\varepsilon}\left(w_{k_{2}}^{0}+\frac{1}{2} \frac{u_{e}^{1 / 2}}{v_{e}^{1 / 2}} \frac{L_{e} / \varepsilon}{\left(i k_{2}+L_{e} / \varepsilon\right)} v_{k_{2}}^{0}\right) \overline{2 \frac{w_{e}^{1 / 2} v_{e}^{1 / 2}}{\varepsilon}\left(u_{k_{1}}^{0}-\frac{1}{2} \frac{w_{e}^{1 / 2}}{v_{e}^{1 / 2}} \frac{L_{e} / \varepsilon}{i k_{1}-L_{e} / \varepsilon} v_{k_{1}}^{0}\right)}\right\}\right\}= \\
& =\sum_{\substack{k_{1}+k_{2}=0, k_{1}, k_{2} \in \mathbb{Z}_{0}}}\left\{\overline{\left(w_{k_{2}}^{0}+\frac{L_{e} / \varepsilon}{\left(i k_{2}+L_{e} / \varepsilon\right)} \frac{1}{2} \frac{u_{e}^{1 / 2}}{v_{e}^{1 / 2}} v_{k_{2}}^{0}\right)}\left(u_{k_{1}}^{0}-\frac{L_{e} / \varepsilon}{\left(i k_{1}-L_{e} / \varepsilon\right)} \frac{1}{2} \frac{w_{e}^{1 / 2}}{v_{e}^{1 / 2}} v_{k_{1}}^{0}\right)+\right. \\
& \left.+\overline{\left(u_{k_{1}}^{0}-\frac{L_{e} / \varepsilon}{\left(i k_{1}-L_{e} / \varepsilon\right)} \frac{1}{2} \frac{w_{e}^{1 / 2}}{v_{e}^{1 / 2}} v_{k_{1}}^{0}\right)}\left(w_{k_{2}}^{0}+\frac{L_{e} / \varepsilon}{\left(i k_{2}+L_{e} / \varepsilon\right)} \frac{1}{2} \frac{u_{e}^{1 / 2}}{v_{e}^{1 / 2}} v_{k_{2}}^{0}\right)\right\}
\end{aligned}
$$

Вычитаем из $D_{0}$ :

$$
\begin{aligned}
& \mathcal{D}_{0}\left(Q_{m}^{+}, Q_{m}^{-}\right)=D_{0}-D_{0}^{(1)}= \\
& =\sum_{\substack{k_{1}+k_{2}=0, k_{1}, k_{2} \in \mathbb{Z}_{0}}}\left\{\overline{\left(w_{k_{2}}^{0}+\frac{L_{e} / \varepsilon}{\left(i k_{2}+L_{e} / \varepsilon\right)} \frac{1}{2} \frac{u_{e}^{1 / 2}}{v_{e}^{1 / 2}} v_{k_{2}}^{0}\right)}\left(u_{k_{1}}^{0}-\frac{L_{e} / \varepsilon}{\left(i k_{1}-L_{e} / \varepsilon\right)} \frac{1}{2} \frac{w_{e}^{1 / 2}}{v_{e}^{1 / 2}} v_{k_{1}}^{0}\right)-\right. \\
& \left.-\overline{\left(u_{k_{1}}^{0}-\frac{L_{e} / \varepsilon}{\left(i k_{1}-L_{e} / \varepsilon\right)} \frac{1}{2} \frac{w_{e}^{1 / 2}}{v_{e}^{1 / 2}} v_{k_{1}}^{0}\right)}\left(w_{k_{2}}^{0}+\frac{L_{e} / \varepsilon}{\left(i k_{2}+L_{e} / \varepsilon\right)} \frac{1}{2} \frac{u_{e}^{1 / 2}}{v_{e}^{1 / 2}} v_{k_{2}}^{0}\right)\right\}-
\end{aligned}
$$




$$
\begin{aligned}
& -\sum_{\substack{k_{1}+k_{2}=0, k_{1}, k_{2} \in \mathbb{Z}_{0}}}\left\{\overline{\left(w_{k_{2}}^{0}+\frac{L_{e} / \varepsilon}{\left(i k_{2}+L_{e} / \varepsilon\right)} \frac{1}{2} \frac{u_{e}^{1 / 2}}{v_{e}^{1 / 2}} v_{k_{2}}^{0}\right)}\left(u_{k_{1}}^{0}-\frac{L_{e} / \varepsilon}{\left(i k_{1}-L_{e} / \varepsilon\right)} \frac{1}{2} \frac{w_{e}^{1 / 2}}{v_{e}^{1 / 2}} v_{k_{1}}^{0}\right)-\right. \\
& \left.-\overline{\left(u_{k_{1}}^{0}-\frac{L_{e} / \varepsilon}{\left(i k_{1}-L_{e} / \varepsilon\right)} \frac{1}{2} \frac{w_{e}^{1 / 2}}{v_{e}^{1 / 2}} v_{k_{1}}^{0}\right)}\left(w_{k_{2}}^{0}+\frac{L_{e} / \varepsilon}{\left(i k_{2}+L_{e} / \varepsilon\right)} \frac{1}{2} \frac{u_{e}^{1 / 2}}{v_{e}^{1 / 2}} v_{k_{2}}^{0}\right)\right\}=0 .
\end{aligned}
$$

Таким образом, $Q_{m}^{+}$и $Q_{m}^{-}$есть решение уравнения секулярности нулевой моды:

$$
\mathcal{D}_{0}\left(Q_{m}^{+}, Q_{m}^{-}\right)=0
$$

5. Оценки линеаризованного оператора в банаховом пространсве $L_{2, \gamma}\left(\mathbb{R}_{+} ; \mathcal{H}_{\sigma}^{(m)}\right)$. Для доказательства теоремы существования и единственности решения системы нелинейных уравнений (9) нам понадобятся следующие оценки линеаризованного оператора $T_{k}^{-1}\left(z_{k}^{(m)}\right)$ в $L_{2, \gamma}\left(\mathbb{R}_{+} ; \mathcal{H}_{\sigma}^{(m)}\right)$ (доказательства см. в [12]).

Лемма 1. Пусть $0<\mu_{0}<1$. Тогда существует такая константа $c_{0}>0$, не зависящая от $\varepsilon, m$, что для любых $k \in \mathbb{Z}_{0}$ имеем

$$
\left\|T^{-1}\left(Z^{(m)}\right)\right\|_{L_{2, \gamma}\left(\mathbb{R}_{+} ; \mathcal{H}_{\sigma}^{(m)}\right)} \leqslant c_{0}\left\|Z^{(m)}\right\|_{L_{2, \gamma}\left(\mathbb{R}_{+} ; \mathcal{H}_{\sigma}^{(m)}\right)^{\circ}}
$$

Более того, для $c_{1}>0$ имеем

$$
\left\|\frac{d}{d t} T^{-1}\left(Z^{(m)}\right)\right\|_{L_{2, \gamma}\left(\mathbb{R}_{+} ; \mathcal{H}_{\sigma}^{(m)}\right)} \leqslant c_{1}\left\|Z^{(m)}\right\|_{L_{2, \gamma}\left(\mathbb{R}_{+} ; \mathcal{H}_{\sigma}^{(m)}\right)} .
$$

Теперь положим

$$
\begin{gathered}
I_{k}^{1}\left(T_{k}^{-1}\left(z_{k}\right)\right)=-i \int_{0}^{t} e^{i k(s-t)} T_{k}^{-1}\left(z_{k}\right) d s, \quad I_{k}^{2}\left(T_{k}^{-1}\left(z_{k}\right)\right)=i \int_{0}^{t} e^{-i k(s-t)} T_{k}^{-1}\left(z_{k}\right) d s, \\
I^{j}\left(T^{-1}(Z)\right)=\left\{I_{k}^{j}\left(T_{k}^{-1}\left(z_{k}\right)\right), \quad k \in \mathbb{Z}_{0}\right\}, \quad \mathcal{I}^{j}\left(T^{-1}(Z)\right)=\left\{k I_{k}^{j}\left(T_{k}^{-1}\left(z_{k}\right)\right), \quad k \in \mathbb{Z}_{0}\right\}, \quad j=1,2 .
\end{gathered}
$$

Лемма 2. Существует такал константа $c_{2}>0$ не зависящая от $\varepsilon, m$, что для любых $k \in \mathbb{Z}_{0} u \gamma=\varepsilon \mu_{0}>0$ имеем

$$
\left\|\mathcal{I}^{j}\left(T^{-1}\left(Z^{(m)}\right)\right)\right\|_{L_{2, \gamma}\left(\mathbb{R}_{+} ; \mathcal{H}_{\sigma}^{(m)}\right)} \leqslant c_{2}\left\|Z^{(m)}\right\|_{L_{2, \gamma}\left(\mathbb{R}_{+} ; \mathcal{H}_{\sigma}^{(m)}\right)^{\prime}}
$$

В тоже время

$$
\left\|I^{j}\left(T^{-1}\left(Z^{(m)}\right)\right)\right\|_{L_{2, \gamma}\left(\mathbb{R}_{+} ; \mathcal{H}_{\sigma}^{(m)}\right)} \leqslant c_{3}\left\|Z^{(m)}\right\|_{L_{2, \gamma}\left(\mathbb{R}_{+} ; \mathcal{H}_{\sigma}^{(m)}\right)} .
$$

Далее, определим класс решений следующей задачи Коши:

$$
T_{k}\left(z_{k}^{(m)}\right)=\frac{d}{d t} z_{k}^{(m)}+\frac{1}{\varepsilon} L_{e} z_{k}^{(m)}+\frac{i k}{\varepsilon} \int_{0}^{t}\left(u_{e} e^{-i k(s-t)}-w_{e} e^{i k(s-t)}\right) z_{k}^{(m)} d s=e^{-i k t},\left.\quad z_{k}^{(m)}\right|_{t=0}=0 .
$$

Лемма 3. Существует такал константа $c_{4}>0$, не зависящая от $\varepsilon, m$, что для любых $k \in \mathbb{Z}_{0}$ u $\gamma=\varepsilon \mu_{0}, \mu_{0}>0$, решение задачи Коши (18) $Z_{\text {sol }}^{-,(m)}=\left\{T_{k}^{-1}\left(e^{-i k t}\right), k \in \mathbb{Z}_{0},|k| \leqslant m\right\}$ удовлетворяет условиям

$$
Z_{\text {sol }}^{-,(m)} \in L_{2, \gamma}\left(\mathbb{R}_{+}\right), \quad \gamma=\varepsilon \mu_{0}, \quad\left\|Z_{\text {sol }}^{-,(m)}\right\|_{L_{2, \gamma}\left(\mathbb{R}_{+}\right)} \leqslant c_{4} \quad \forall k \in \mathbb{Z}_{0} .
$$

Более того, для любой $Q_{m}^{-}=\left\{Q_{k, m}^{-}, k \in \mathbb{Z}_{0},|k| \leqslant m\right\} \in \mathcal{H}_{\sigma}^{(m)}$ имеют место следующие оценки:

$$
\left\|Q_{m}^{-} Z_{\mathrm{sol}}^{-,(m)}\right\|_{L_{2, \gamma}\left(\mathbb{R}_{+} ; \mathcal{H}_{\sigma}^{(m)}\right)} \leqslant c_{5}\left\|Q_{m}^{-}\right\|_{\mathcal{H}_{\sigma}^{(m)}}, \quad\left\|\frac{d}{d t} Q_{m}^{-} Z_{s o l}^{-,(m)}\right\|_{L_{2, \gamma}\left(\mathbb{R}_{+} ; \mathcal{H}_{\sigma}^{(m)}\right)} \leqslant c_{6}\left\|Q_{m}^{-}\right\|_{\mathcal{H}_{\sigma}^{(m)}} .
$$


Аналогично определяем решение задачи Коши

$$
T_{k}\left(z_{k}^{(m)}\right)=\frac{d}{d t} z_{k}^{(m)}+\frac{1}{\varepsilon} L_{e} z_{k}^{(m)}+\frac{i k}{\varepsilon} \int_{0}^{t}\left(u_{e} e^{-i k(s-t)}-w_{e} e^{i k(s-t)}\right) z_{k}^{(m)} d s=e^{i k t},\left.\quad z_{k}^{(m)}\right|_{t=0}=0 .
$$

Лемма 4. Существует такая константа $c_{7}>0$, не зависящая от $\varepsilon, m$, что для любых $k \in \mathbb{Z}_{0}$ и $\gamma=\varepsilon \mu_{0}, \mu_{0}>0$, решение задачи Коши (19) $Z_{\text {sol }}^{+,(m)}=\left\{T_{k}^{-1}\left(e^{i k t}\right), k \in \mathbb{Z}_{0},|k| \leqslant m\right\}$ удовлетворяет условиям

$$
Z_{\text {sol }}^{+,(m)} \in L_{2, \gamma}\left(\mathbb{R}_{+}\right), \quad \gamma=\varepsilon \mu_{0}, \quad\left\|Z_{\text {sol }}^{+,(m)}\right\|_{L_{2, \gamma}\left(\mathbb{R}_{+}\right)} \leqslant c_{7} \quad \forall k \in \mathbb{Z}_{0} .
$$

Более того, для любой $Q_{m}^{+}=\left\{Q_{k, m}^{+}, k \in \mathbb{Z}_{0},|k| \leqslant m\right\} \in \mathcal{H}_{\sigma}^{(m)}$ имеют место следующие оценки:

$$
\left\|Q_{m}^{+} Z_{\text {sol }}^{+,(m)}\right\|_{L_{2, \gamma}\left(\mathbb{R}_{+} ; \mathcal{H}_{\sigma}^{(m)}\right)} \leqslant c_{8}\left\|Q_{m}^{+}\right\|_{\mathcal{H}_{\sigma}^{(m)}}, \quad\left\|\frac{d}{d t} Q_{m}^{+} Z_{\text {sol }}^{+,(m)}\right\|_{L_{2, \gamma}\left(\mathbb{R}_{+} ; \mathcal{H}_{\sigma}^{(m)}\right)} \leqslant c_{9}\left\|Q_{m}^{+}\right\|_{\mathcal{H}_{\sigma}^{(m)}} .
$$

\section{6. Оценки правой части нелинейного уравнения.}

Лемма 5. Для $\sigma>2$ справедливы следующие оценки:

$$
\begin{aligned}
& \varepsilon w_{e}^{1 / 2}\left\|B^{(m)}\left(T^{-1}\left(Z^{(m)}\right), T^{-1}\left(Z^{(m)}\right)\right)\right\|_{L_{2, \gamma}\left(\mathbb{R}_{+} ; \mathcal{H}_{\sigma}^{(m)}\right)} \leqslant c_{1, \sigma}\left\|Z^{(m)}\right\|_{L_{2, \gamma}\left(\mathbb{R}_{+} ; \mathcal{H}_{\sigma}^{(m)}\right)^{2}}^{2} \\
& \varepsilon w_{e}^{1 / 2}\left\|L^{(m)}\left(T^{-1}\left(Z^{(m)}\right)\right)\right\|_{L_{2, \gamma}\left(\mathbb{R}_{+} ; \mathcal{H}_{\sigma}^{(m)}\right)}^{2} \leqslant \\
& \leqslant\left(\left\|u^{0}\right\|\left\|_{\mathcal{H}_{\sigma}^{(m)}}+\right\| v^{0}\|\|_{\mathcal{H}_{\sigma}^{(m)}}+\|\| w^{0} \|_{\mathcal{H}_{\sigma}^{(m)}}\right) c_{2, \sigma}\left\|Z^{(m)}\right\|_{L_{2, \gamma}\left(\mathbb{R}_{+} ; \mathcal{H}_{\sigma}^{(m)}\right)}, \\
& \varepsilon w_{e}^{1 / 2}\left\|B^{(m)}\left(T^{-1}\left(Z^{(m)}\right), T^{-1}\left(D^{(m)}\right)\right)\right\|_{L_{2, \gamma}\left(\mathbb{R}_{+} ; \mathcal{H}_{\sigma}^{(m)}\right)} \leqslant \\
& \leqslant \frac{1}{\sqrt{\varepsilon}}\left(\left\|u^{0}\right\|_{\mathcal{H}_{\sigma}^{(m)}}+\left\|v^{0} \mid\right\|_{\mathcal{H}_{\sigma}^{(m)}}+\left\|w^{0}\right\| \|_{\mathcal{H}_{\sigma}^{(m)}}\right) c_{3, \sigma}\left\|Z^{(m)}\right\|_{L_{2, \gamma}\left(\mathbb{R}_{+} ; \mathcal{H}_{\sigma}^{(m)}\right)}, \\
& \varepsilon w_{e}^{1 / 2}\left\|B^{(m)}\left(T^{-1}\left(D^{(m)}\right), T^{-1}\left(Z^{(m)}\right)\right)\right\|_{L_{2, \gamma}\left(\mathbb{R}_{+} ; \mathcal{H}_{\sigma}^{(m)}\right)} \leqslant \\
& \leqslant \frac{1}{\sqrt{\varepsilon}}\left(\left\|u^{0}\right\|_{\mathcal{H}_{\sigma}^{(m)}}+\left\|v^{0} \mid\right\|_{\mathcal{H}_{\sigma}^{(m)}}+\left\|w^{0}\right\| \|_{\mathcal{H}_{\sigma}^{(m)}}\right) c_{4, \sigma}\left\|Z^{(m)}\right\|_{L_{2, \gamma}\left(\mathbb{R}_{+} ; \mathcal{H}_{\sigma}^{(m)}\right)}, \\
& \left\|Q_{m}^{-} \mid\right\|_{\mathcal{H}_{\sigma}^{(m)}} \leqslant \frac{c_{1}}{\varepsilon}\left(\left|\left\|u^{0}\left|\left\|_{\mathcal{H}_{\sigma}^{(m)}}+\right\|\left\|v^{0}\right\|\left\|_{\mathcal{H}_{\sigma}^{(m)}}+\right\|\right| w^{0}\right\|_{\mathcal{H}_{\sigma}^{(m)}}\right),\right. \\
& \left\|Q_{m}^{+} \mid\right\|_{\mathcal{H}_{\sigma}^{(m)}} \leqslant \frac{c_{2}}{\varepsilon}\left(\left|\left\|u^{0}\left|\left\|_{\mathcal{H}_{\sigma}^{(m)}}+\right\|\right| v^{0}\right\|\left\|_{\mathcal{H}_{\sigma}^{(m)}}+\right\| w^{0} \|_{\mathcal{H}_{\sigma}^{(m)}}\right),\right.
\end{aligned}
$$

где

$$
D^{(m)}=\left(D_{k}^{(m)}, k \in \mathbb{Z}_{0},|k| \leqslant m\right), \quad D_{k}^{(m)}=Q_{k, m}^{-} e^{-i k t}+Q_{k, m}^{+} e^{i k t} .
$$

Доказательство. Для билинейной формы имеем

$$
\begin{aligned}
\varepsilon^{2} v_{e}\left\|B^{(m)}\left(T^{-1}\left(Z^{(m)}\right), T^{-1}\left(Z^{(m)}\right)\right)\right\|_{L_{2, \gamma}\left(\mathbb{R}_{+} ; \mathcal{H}_{\sigma}^{(m)}\right)}^{2} \leqslant & \left\{T_{k_{1}}^{-1}\left(z_{k_{1}}^{(m)}\right) \overline{T_{k_{2}}^{-1}\left(z_{k_{2}}^{(m)}\right)}-\right. \\
\leqslant \varepsilon^{2} v_{e} \sum_{\substack{k_{1}+k_{2}=k,\left|k_{1}\right|,\left|k_{2}\right|=1, \ldots, m}} & \left(-\frac{w_{e}^{1 / 2}}{v_{e}^{1 / 2}} T_{k_{1}}^{-1}\left(z_{k_{1}}^{(m)}\right)+i k_{1} \frac{w_{e}^{1 / 2}}{v_{e}^{1 / 2}} \int_{0}^{t} e^{i k_{1}(s-t)} T_{k_{1}}^{-1}\left(z_{k_{1}}^{(m)}\right) d s\right) \times \\
& \left.-\frac{1}{4}\right)
\end{aligned}
$$




$$
\begin{aligned}
& \times \overline{\left.\left(-\frac{u_{e}^{1 / 2}}{v_{e}^{1 / 2}} T_{k_{2}}^{-1}\left(z_{k_{2}}^{(m)}\right)-i k_{2} \frac{u_{e}^{1 / 2}}{v_{e}^{1 / 2}} \int_{0}^{t} e^{-i k_{2}(s-t)} T_{k_{2}}^{-1}\left(z_{k_{2}}^{(m)}\right) d s\right)\right\}} \|_{L_{2, \gamma}\left(\mathbb{R}_{+} ; \mathcal{H}_{\sigma}^{(m)}\right)}^{2}+ \\
&+\varepsilon^{2} v_{e} \| \sum_{\substack{k_{1}+k_{2}=k,\left|k_{1}\right|,\left|k_{2}\right|=1, \ldots, m}}\left\{T _ { k _ { 2 } } ^ { - 1 } \left(z_{k_{2}}^{(m)} \overline{T_{k_{1}}^{-1}\left(z_{k_{1}}^{(m)}\right)}-\right.\right. \\
& \frac{-\frac{1}{4}\left(-\frac{u_{e}^{1 / 2}}{v_{e}^{1 / 2}} T_{k_{2}}^{-1}\left(z_{k_{2}}^{(m)}\right)-i k_{2} \frac{u_{e}^{1 / 2}}{v_{e}^{1 / 2}} \int_{0}^{t} e^{-i k_{2}(s-t)} T_{k_{2}}^{-1}\left(z_{k_{2}}^{(m)}\right) d s\right) \times}{\left.\left(-\frac{w_{e}^{1 / 2}}{v_{e}^{1 / 2}} T_{k_{1}}^{-1}\left(z_{k_{1}}^{(m)}\right)+i k_{1} \frac{w_{e}^{1 / 2}}{v_{e}^{1 / 2}} \int_{0}^{t} e^{i k_{1}(s-t)} T_{k_{1}}^{-1}\left(z_{k_{1}}^{(m)}\right) d s\right)\right\} \|_{L_{2, \gamma}\left(\mathbb{R}_{+} ; \mathcal{H}_{\sigma}^{(m)}\right)}^{2}=B_{1}+B_{2} .}
\end{aligned}
$$

Переходя к оценке для $B_{1}$, имеем

$$
\begin{aligned}
& B_{1}=\varepsilon^{2} v_{e} \int_{0}^{\infty} e^{2 \gamma t} \sup _{\substack{k \in \mathbb{Z}_{0},|k| \leqslant m}}|k|^{2 \sigma} \mid \sum_{\substack{k_{1}+k_{2}=k,\left|k_{1}\right|,\left|k_{2}\right|=1, \ldots, m}}\left\{T_{k_{1}}^{-1}\left(z_{k_{1}}^{(m)}\right) \overline{T_{k_{2}}^{-1}\left(z_{k_{2}}^{(m)}\right)}-\right. \\
& -\frac{1}{4}\left(-\frac{w_{e}^{1 / 2}}{v_{e}^{1 / 2}} T_{k_{1}}^{-1}\left(z_{k_{1}}^{(m)}\right)+i k_{1} \frac{w_{e}^{1 / 2}}{v_{e}^{1 / 2}} \int_{0}^{t} e^{i k_{1}(s-t)} T_{k_{1}}^{-1}\left(z_{k_{1}}^{(m)}\right) d s\right) \times \\
& \left.\times \overline{\left(-\frac{u_{e}^{1 / 2}}{v_{e}^{1 / 2}} T_{k_{2}}^{-1}\left(z_{k_{2}}^{(m)}\right)-i k_{2} \frac{u_{e}^{1 / 2}}{v_{e}^{1 / 2}} \int_{0}^{t} e^{-i k_{2}(s-t)} T_{k_{2}}^{-1}\left(z_{k_{2}}^{(m)}\right) d s\right)}\right\}\left.\right|^{2} d t .
\end{aligned}
$$

Воспользовавшись неравенством

$$
\left(\left|k_{1}\right|+\left|k_{2}\right|\right)^{\sigma} \leqslant c_{\sigma}\left(\left|k_{1}\right|^{\sigma}+\left|k_{2}\right|^{\sigma}\right), c_{\sigma}=2^{\sigma-1},
$$

вносим супремум под знак суммы:

$$
\begin{aligned}
& B_{1} \leqslant \varepsilon^{2} v_{e} c_{\sigma}^{2} \int_{0}^{\infty} e^{2 \gamma t} \mid \sum_{\substack{k_{1}+k_{2}=k,\left|k_{1}\right|,\left|k_{2}\right|=1, \ldots, m}}\left\{\sup _{\substack{k_{1} \in \mathbb{Z}_{0},\left|k_{1}\right| \leqslant m}}\left|k_{1}\right|^{\sigma} T_{k_{1}}^{-1}\left(z_{k_{1}}^{(m)}\right)\left|k_{2}\right|^{\sigma} \frac{1}{\left|k_{2}\right|^{\sigma}} \overline{T_{k_{2}}^{-1}\left(z_{k_{2}}^{(m)}\right)}-\right. \\
& -\frac{1}{4} \sup _{\substack{k_{1} \in \mathbb{Z}_{0},\left|k_{1}\right| \leqslant m}}\left|k_{1}\right|^{\sigma}\left(-\frac{w_{e}^{1 / 2}}{v_{e}^{1 / 2}} T_{k_{1}}^{-1}\left(z_{k_{1}}^{(m)}\right)+i k_{1} \frac{w_{e}^{1 / 2}}{v_{e}^{1 / 2}} \int_{0}^{t} e^{i k_{1}(s-t)} T_{k_{1}}^{-1}\left(z_{k_{1}}^{(m)}\right) d s\right) \times \\
& \left.\times \frac{1}{\left|k_{2}\right|^{\sigma}} \overline{\left(-\frac{u_{e}^{1 / 2}}{v_{e}^{1 / 2}}\left|k_{2}\right|^{\sigma} T_{k_{2}}^{-1}\left(z_{k_{2}}^{(m)}\right)-i k_{2} \frac{u_{e}^{1 / 2}}{v_{e}^{1 / 2}} \int_{0}^{t} e^{-i k_{2}(s-t)}\left|k_{2}\right|^{\sigma} T_{k_{2}}^{-1}\left(z_{k_{2}}^{(m)}\right) d s\right)}\right\}\left.\right|^{2} d t+ \\
& +\varepsilon^{2} v_{e} c_{\sigma}^{2} \int_{0}^{\infty} e^{2 \gamma t} \mid \sum_{\substack{k_{1}+k_{2}=k,\left|k_{1}\right|,\left|k_{2}\right|=1, \ldots, m}}\left\{\frac{\left|k_{1}\right|^{\sigma}}{\left|k_{1}\right|^{\sigma}} T_{k_{1}}^{-1}\left(z_{k_{1}}^{(m)}\right) \sup _{\substack{k_{2} \in \mathbb{Z}_{0},\left|k_{2}\right| \leqslant m}}\left|k_{2}\right|^{\sigma} \overline{T_{k_{2}}^{-1}\left(z_{k_{2}}^{(m)}\right)}-\right. \\
& -\frac{1}{4} \frac{1}{\left|k_{1}\right|^{\sigma}}\left(-\frac{w_{e}^{1 / 2}}{v_{e}^{1 / 2}}\left|k_{1}\right|^{\sigma} T_{k_{1}}^{-1}\left(z_{k_{1}}^{(m)}\right)+i k_{1} \frac{w_{e}^{1 / 2}}{v_{e}^{1 / 2}} \int_{0}^{t} e^{i k_{1}(s-t)}\left|k_{1}\right|^{\sigma} T_{k_{1}}^{-1}\left(z_{k_{1}}^{(m)}\right) d s\right) \times
\end{aligned}
$$




$$
\left.\times \sup _{\substack{k_{2} \in \mathbb{Z}_{0} \\\left|k_{2}\right| \leqslant m}} \overline{\left(-\frac{u_{e}^{1 / 2}}{v_{e}^{1 / 2}}\left|k_{2}\right|^{\sigma} T_{k_{2}}^{-1}\left(z_{k_{2}}^{(m)}\right)-i k_{2} \frac{u_{e}^{1 / 2}}{v_{e}^{1 / 2}} \int_{0}^{t} e^{-i k_{2}(s-t)}\left|k_{2}\right|^{\sigma} T_{k_{2}}^{-1}\left(z_{k_{2}}^{(m)}\right) d s\right)}\right\}\left.\right|^{2} d t .
$$

Введем обозначения

$$
\begin{gathered}
A_{1}=T_{k_{1}}^{-1}\left(z_{k_{1}}^{(m)}\right) \frac{T_{k_{2}}^{-1}\left(z_{k_{2}}^{(m)}\right), \quad A_{2}=-T_{k_{1}}^{-1}\left(z_{k_{1}}^{(m)}\right)+i k_{1} \int_{0}^{t} e^{i k_{1}(s-t)} T_{k_{1}}^{-1}\left(z_{k_{1}}^{(m)}\right) d s,}{A_{3}=-T_{k_{2}}^{-1}\left(z_{k_{2}}^{(m)}\right)-i k_{2} \int_{0}^{t} e^{-i k_{2}(s-t)} T_{k_{2}}^{-1}\left(z_{k_{2}}^{(m)}\right) d s .} \\
\text {. }
\end{gathered}
$$

С учетом этих обозначений $B_{1}$ примет вид

$$
\begin{aligned}
& B_{1} \leqslant \varepsilon^{2} v_{e} c_{\sigma}^{2} \int_{0}^{\infty} e^{2 \gamma t}\left|\sum_{\substack{k_{1}+k_{2}=k,\left|k_{1}\right|,\left|k_{2}\right|=1, \ldots, m}}\left\{\sup _{\substack{k_{1} \in \mathbb{Z}_{0},\left|k_{1}\right| \leqslant m}}\left|k_{1}\right|^{\sigma}\left|k_{2}\right|^{\sigma} \frac{1}{\left|k_{2}\right|^{\sigma}} A_{1}-\frac{1}{4} \sup _{\substack{k_{1} \in \mathbb{Z}_{0},\left|k_{1}\right| \leqslant m}}\left|k_{1}\right|^{\sigma} \frac{1}{\left|k_{2}\right|^{\sigma}}\left|k_{2}\right|^{\sigma} A_{2} A_{3}\right\}\right|^{2} d t+ \\
& +\varepsilon^{2} v_{e} c_{\sigma}^{2} \int_{0}^{\infty} e^{2 \gamma t}\left|\sum_{\substack{k_{1}+k_{2}=k,\left|k_{1}\right|,\left|k_{2}\right|=1, \ldots, m}}\left\{\left|k_{1}\right|^{\sigma} \frac{1}{\left|k_{1}\right|^{\sigma}} \sup _{\substack{k_{2} \in \mathbb{Z}_{0},\left|k_{2}\right| \leqslant m}}\left|k_{2}\right|^{\sigma} A_{1}-\frac{1}{4} \frac{1}{\left|k_{1}\right|^{\sigma}}\left|k_{1}\right|^{\sigma} \sup _{\substack{k_{2} \in \mathbb{Z}_{0},\left|k_{2}\right| \leqslant m}}\left|k_{2}\right|^{\sigma} A_{2} A_{3}\right\}\right|^{2} d t .
\end{aligned}
$$

Имеем

$$
\begin{aligned}
& R=\left.\left.\varepsilon^{2} v_{e} c_{\sigma}^{2} \int_{0}^{\infty} e^{2 \gamma t}\left|\sum_{\substack{k_{1}+k_{2}=k,\left|k_{1}\right|,\left|k_{2}\right|=1, \ldots, m}} \sup _{\substack{k_{1} \in k_{1}|\leqslant m\\
| \mathbb{Z}_{0},}}\right| k_{1}\right|^{\sigma}\left|k_{2}\right|^{\sigma} \frac{1}{\left|k_{2}\right|^{\sigma}} A_{1}\right|^{2}= \\
& =\left.\left.\varepsilon^{2} v_{e} c_{\sigma}^{2} \int_{0}^{\infty} e^{2 \gamma t}\left|\sum_{\substack{k_{1}+k_{2}=k,\left|k_{1}\right|,\left|k_{2}\right|=1, \ldots, m}} \sup _{\substack{k_{1} \in \mathbb{Z}_{0}|\leqslant m\\
| k_{1} \mid \leqslant m}}\right| k_{1}\right|^{\sigma}\left|k_{2}\right|^{\sigma} \frac{1}{\left|k_{2}\right|^{\sigma}} T_{k_{1}}^{-1}\left(z_{k_{1}}^{(m)}\right) \overline{T_{k_{2}}^{-1}\left(z_{k_{2}}^{(m)}\right)}\right|^{2} \leqslant \\
& \leqslant\left.\left. c_{\sigma}^{2} \sup _{t \in \mathbb{R}_{+} \sup _{2} \in \mathbb{Z}_{0},} \operatorname{su}_{\left|k_{2}\right| \leqslant m}\left|k_{2}\right|^{2 \sigma}\left|\overline{T_{k_{2}}^{-1}\left(z_{k_{2}}^{(m)}\right)}\right|^{2} \varepsilon^{2} v_{e} \int_{0}^{\infty} e^{2 \gamma t}\left|\sup _{\substack{k_{1} \in \mathbb{Z}_{0},\left|k_{1}\right| \leqslant m}}\right| k_{1}\right|^{\sigma} T_{k_{1}}^{-1}\left(z_{k_{1}}^{(m)}\right)\right|^{2}\left(\sum_{k_{2}=1}^{\infty} \frac{1}{\left|k_{2}\right|^{\sigma}}\right)^{2} .
\end{aligned}
$$

Для супремума имеем оценку

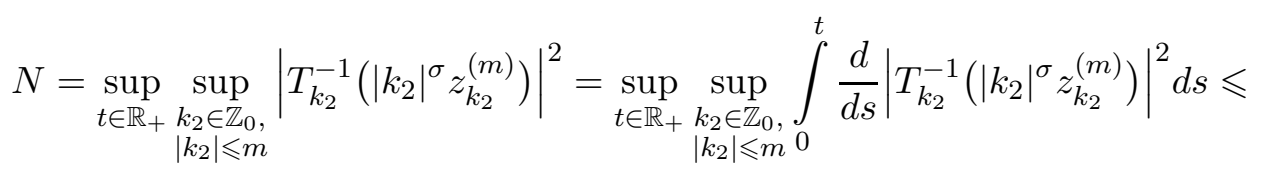

$$
\begin{aligned}
& \leqslant 2 \sup _{t \in \mathbb{R}_{+}} \sup _{\substack{k_{2} \in \mathbb{Z}_{0},\left|k_{2}\right| \leqslant m}} \int_{0}^{t}\left|T_{k_{2}}^{-1}\left(\left|k_{2}\right|^{\sigma} z_{k_{2}}^{(m)}\right)\right| \cdot\left|\frac{d}{d s} T_{k_{2}}^{-1}\left(\left|k_{2}\right|^{\sigma} z_{k_{2}}^{(m)}\right)\right| d s .
\end{aligned}
$$

Используя неравенство Гельдера, получим

$$
N \leqslant 2\left(\int_{0}^{\infty} \sup _{\substack{k_{2} \in \mathbb{Z}_{0},\left|k_{2}\right| \leqslant m}}\left|T_{k_{2}}^{-1}\left(\left|k_{2}\right|^{\sigma} z_{k_{2}}^{(m)}\right)\right|^{2} d s\right)^{1 / 2}\left(\int_{\substack{0 \\ 0}}^{\infty} \sup _{\substack{k_{2} \in \mathbb{Z}_{0},\left|k_{2}\right| \leqslant m}}\left|\frac{d}{d s} T_{k_{2}}^{-1}\left(\left|k_{2}\right|^{\sigma} z_{k_{2}}^{(m)}\right)\right|^{2} d s\right)^{1 / 2} .
$$

Заметим, что $1 \leqslant e^{2 \gamma t}, t \geqslant 0$. Значит, можно переписать неравенство в виде 


$$
\begin{array}{r}
N \leqslant 2 \sup _{\substack{k_{2} \in \mathbb{Z}_{0},\left|k_{2}\right| \leqslant m}}\left(\int_{0}^{\infty} e^{2 \gamma s} \sup _{\substack{k_{2} \in \mathbb{Z}_{0},\left|k_{2}\right| \leqslant m}}\left|T_{k_{2}}^{-1}\left(\left|k_{2}\right|^{\sigma} z_{k_{2}}^{(m)}\right)\right|^{2} d s\right)^{1 / 2}\left(\int_{0}^{\infty} e^{2 \gamma s} \sup _{\substack{k_{2} \in \mathbb{Z}_{0},\left|k_{2}\right| \leqslant m}}\left|\frac{d}{d s} T_{k_{2}}^{-1}\left(\left|k_{2}\right|^{\sigma} z_{k_{2}}^{(m)}\right)\right|^{2} d s\right)^{1 / 2}= \\
=2\left\|X_{2}^{(m)}\right\|_{L_{2, \gamma}\left(\mathbb{R}_{+} ; \mathcal{H}_{\sigma}^{(m)}\right)}\left\|\frac{d}{d t} X_{2}^{(m)}\right\|_{L_{2, \gamma}\left(\mathbb{R}_{+} ; \mathcal{H}_{\sigma}^{(m)}\right)} .
\end{array}
$$

Отсюда

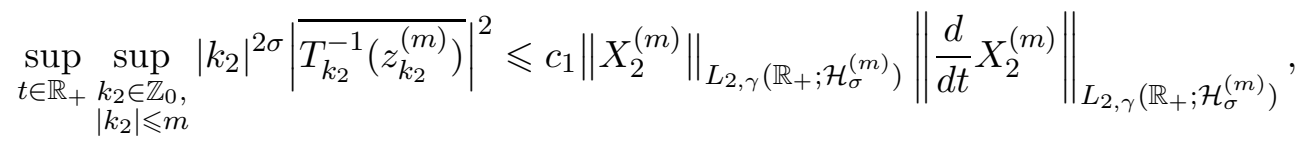

где

$$
X_{2}^{(m)}=\left\{T_{k_{2}}^{-1}\left(z_{k_{2}}^{(m)}\right),\left|k_{2}\right| \leqslant m, k_{2} \in \mathbb{Z}_{0}\right\} .
$$

После этого воспользуемся неравенствами $(14),(15)$, в силу которых имеем

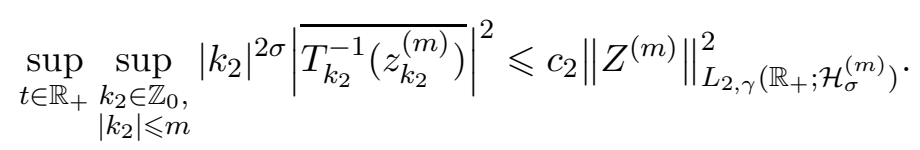

Далее,

$$
\left.\left.\int_{0}^{\infty} e^{2 \gamma t}\left|\sup _{\substack{k_{1} \in \mathbb{Z}_{0},\left|k_{1}\right| \leqslant m}}\right| k_{1}\right|^{\sigma} T_{k_{1}}^{-1}\left(z_{k_{1}}^{(m)}\right)\right|^{2}=\left\|X_{1}^{(m)}\right\|_{L_{2, \gamma}\left(\mathbb{R}_{+} ; \mathcal{H}_{\sigma}^{(m)}\right)}^{2} \leqslant c_{3}^{2}\left\|Z^{(m)}\right\|_{L_{2, \gamma}\left(\mathbb{R}_{+} ; \mathcal{H}_{\sigma}^{(m)}\right)}^{2}
$$

где

В итоге получаем

$$
X_{1}^{(m)}=\left\{T_{k_{1}}^{-1}\left(z_{k_{1}}^{(m)}\right),\left|k_{1}\right| \leqslant m, k_{1} \in \mathbb{Z}_{0}\right\} .
$$

$$
R \leqslant c_{4, \sigma}^{2}\left\|Z^{(m)}\right\|_{L_{2, \gamma}\left(\mathbb{R}_{+} ; \mathcal{H}_{\sigma}^{(m)}\right)}^{4} .
$$

Переходим к следующему выражению:

$$
\begin{aligned}
& \left.\left.\varepsilon^{2} v_{e} c_{\sigma}^{2} \int_{0}^{\infty} e^{2 \gamma t}\left|\sum_{\substack{k_{1}+k_{2}=k,\left|k_{1}\right|,\left|k_{2}\right|=1, \ldots, m}} \frac{1}{4} \sup _{\substack{k_{1} \in \mathbb{Z}_{0},\left|k_{1}\right| \leqslant m}} \frac{\left|k_{1}\right|^{\sigma}}{\left|k_{2}\right|^{\sigma}}\right| k_{2}\right|^{\sigma} A_{2} A_{3}\right|^{2} d t= \\
& =\left.\varepsilon^{2} v_{e} c_{\sigma}^{2} \int_{0}^{\infty} e^{2 \gamma t}\left|\sum_{\substack{k_{1}+k_{2}=k,\left|k_{1}\right|,\left|k_{2}\right|=1, \ldots, m}} \frac{1}{4} \sup _{\substack{k_{1} \in \mathbb{Z}_{0},\left|k_{1}\right| \leqslant m}} \frac{\left|k_{1}\right|^{\sigma}}{\left|k_{2}\right|^{\sigma}}\right| k_{2}\right|^{\sigma}\left(-T_{k_{1}}^{-1}\left(z_{k_{1}}^{(m)}\right)+i k_{1} \int_{0}^{t} e^{i k_{1}(s-t)} T_{k_{1}}^{-1}\left(z_{k_{1}}^{(m)}\right) d s\right) \times \\
& \times\left.\overline{\left(-T_{k_{2}}^{-1}\left(z_{k_{2}}^{(m)}\right)-i k_{2} \int_{0}^{t} e^{-i k_{2}(s-t)} T_{k_{2}}^{-1}\left(z_{k_{2}}^{(m)}\right) d s\right)}\right|^{2} d t \leqslant \\
& \leqslant\left.\left.\varepsilon^{2} v_{e} c_{\sigma}^{2} \int_{0}^{\infty} e^{2 \gamma t}\left|\sum_{\substack{k_{1}+k_{2}=k,\left|k_{1}\right|,\left|k_{2}\right|=1, \ldots, m}} \frac{1}{4} \sup _{\substack{k_{1} \in \mathbb{Z}_{0},\left|k_{1}\right| \leqslant m}} \frac{\left|k_{1}\right|^{\sigma}}{\left|k_{2}\right|^{\sigma}}\right| k_{2}\right|^{\sigma} T_{k_{1}}^{-1}\left(z_{k_{1}}^{(m)}\right) \overline{T_{k_{2}}^{-1}\left(z_{k_{2}}^{(m)}\right)}\right|^{2} d t+ \\
& +\left.\left.\varepsilon^{2} v_{e} c_{\sigma}^{2} \int_{0}^{\infty} e^{2 \gamma t}\left|\sum_{\substack{k_{1}+k_{2}=k,\left|k_{1}\right|,\left|k_{2}\right|=1, \ldots, m}} \frac{1}{4} \sup _{\substack{k_{1} \in \mathbb{Z}_{0},\left|k_{1}\right| \leqslant m}} \frac{\left|k_{1}\right|^{\sigma}}{\left|k_{2}\right|^{\sigma}}\right| k_{2}\right|^{\sigma} T_{k_{1}}^{-1}\left(z_{k_{1}}^{(m)}\right) i k_{2} \int_{0}^{t} e^{-i k_{2}(s-t)} T_{k_{2}}^{-1}\left(z_{k_{2}}^{(m)}\right) d s\right|^{2} d t+ \\
& +\left.\left.\varepsilon^{2} v_{e} c_{\sigma}^{2} \int_{0}^{\infty} e^{2 \gamma t}\left|\sum_{\substack{k_{1}+k_{2}=k,\left|k_{1}\right|,\left|k_{2}\right|=1, \ldots, m}} \frac{1}{4} \sup _{\substack{k_{1} \in \mathbb{Z}_{0} \\
\left|k_{1}\right| \leqslant m}} \frac{\left|k_{1}\right|^{\sigma}}{\left|k_{2}\right|^{\sigma}}\right| k_{2}\right|^{\sigma} \overline{T_{k_{2}}^{-1}\left(z_{k_{2}}^{(m)}\right)} i k_{1} \int_{0}^{t} e^{i k_{1}(s-t)} T_{k_{1}}^{-1}\left(z_{k_{1}}^{(m)}\right) d s\right|^{2} d t+
\end{aligned}
$$




$$
\begin{aligned}
+\varepsilon^{2} v_{e} c_{\sigma}^{2} \int_{0}^{\infty} e^{2 \gamma t} \mid \sum_{\substack{k_{1}+k_{2}=k,\left|k_{1}\right|,\left|k_{2}\right|=1, \ldots, m}} \frac{\left.\left.\frac{1}{4} \sup _{\substack{k_{1} \in \mathbb{Z}_{0},\left|k_{1}\right| \leqslant m}} \frac{\left|k_{1}\right|^{\sigma}\left|k_{2}\right|^{\sigma}}{k_{2}}\right|_{2}\right|^{\sigma} i k_{1} \int_{0}^{t} e^{i k_{1}(s-t)} T_{k_{1}}^{-1}\left(z_{k_{1}}^{(m)}\right) d s \times}{\left.\left(-i k_{2} \int_{0}^{t} e^{-i k_{2}(s-t)} T_{k_{2}}^{-1}\left(z_{k_{2}}^{(m)}\right) d s\right)\right|^{2}} d t=R_{1}+R_{2}+R_{3}+R_{4} .
\end{aligned}
$$

Первое слагаемое оценивается, как было сделано выше в (22):

$$
R_{1} \leqslant c_{2, \sigma}^{2}\left\|Z^{(m)}\right\|_{L_{2, \gamma}\left(\mathbb{R}_{+} ; \mathcal{H}_{\sigma}^{(m)}\right)}^{4} .
$$

Далее,

$$
\begin{aligned}
& R_{2}=\varepsilon^{2} v_{e} c_{\sigma}^{2} \int_{0}^{\infty} e^{2 \gamma t} \mid \sum_{\substack{k_{1}+k_{2}=k,\left|k_{1}\right|,\left|k_{2}\right|=1, \ldots, m}} \frac{1}{4} \sup _{\substack{k_{1} \in \mathbb{Z}_{0},\left.k_{1}|\leqslant m\\
| k_{2}\right|^{\sigma}}} \frac{\left|k_{1}\right|^{\sigma}}{\left.\left.\left|k_{2}\right| k_{2}\right|^{\sigma} T_{k_{1}}^{-1}\left(z_{k_{1}}^{(m)}\right) i k_{2} \int_{0}^{t} e^{-i k_{2}(s-t)} T_{k_{2}}^{-1}\left(z_{k_{2}}^{(m)}\right) d s\right|^{2}} d t \leqslant \\
& \leqslant\left.\left.\varepsilon^{2} c_{1} \sup _{t} \sup _{\substack{k_{2} \in \mathbb{Z}_{0} \\
\left|k_{2}\right| \leqslant m}}\left|i \int_{0}^{t} e^{-i k_{2}(s-t)\left|k_{2}\right| \sigma T_{k_{2}}^{-1}\left(z_{k_{2}}^{(m)}\right) d s \mid}\right|_{0}^{2} e^{2 \gamma t}\left|\sup _{\substack{k_{1} \in \mathbb{Z}_{0},\left|k_{1}\right| \leqslant m}}^{\infty}\right| k_{1}\right|^{\sigma} T_{k_{1}}^{-1}\left(z_{k_{1}}^{(m)}\right)\right|^{2} d t\left(\sum_{k_{2}=1}^{\infty} \frac{k_{2}}{\left|k_{2}\right|^{\sigma}}\right)^{2} .
\end{aligned}
$$

Здесь для вычисления супремума применяем неравенство Гельдера и оценку (14):

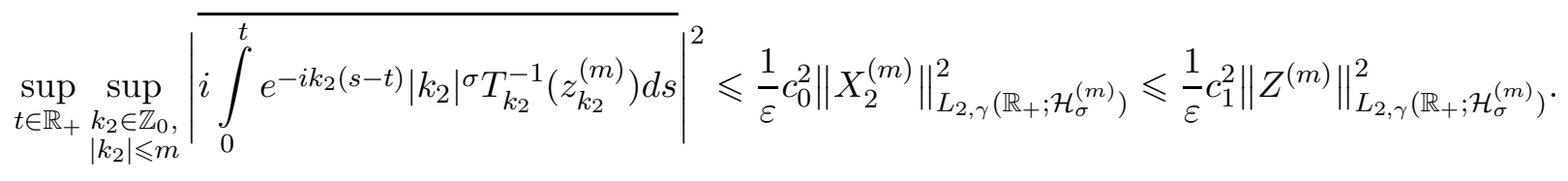

Отсюда получаем неравенство

$$
R_{2} \leqslant \varepsilon c_{3, \sigma}^{2}\left\|Z^{(m)}\right\|_{L_{2, \gamma}\left(\mathbb{R}_{+} ; \mathcal{H}_{\sigma}^{(m)}\right)}^{4} .
$$

Аналогично находим оценки для $R_{3}, R_{4}$ :

$$
R_{3} \leqslant \varepsilon c_{4, \sigma}^{2}\left\|Z^{(m)}\right\|_{L_{2, \gamma}\left(\mathbb{R}_{+} ; \mathcal{H}_{\sigma}^{(m)}\right)}^{4}, \quad R_{4} \leqslant \varepsilon c_{5, \sigma}^{2}\left\|Z^{(m)}\right\|_{L_{2, \gamma}\left(\mathbb{R}_{+} ; \mathcal{H}_{\sigma}^{(m)}\right)}^{4} .
$$

Применяя полученные оценки к (20), приходим к неравенству

$$
B_{1} \leqslant c_{6, \sigma}^{2}\left\|Z^{(m)}\right\|_{L_{2, \gamma}\left(\mathbb{R}_{+} ; \mathcal{H}_{\sigma}^{(m)}\right)}^{4} .
$$

Также получим

Таким образом, имеем

$$
B_{2} \leqslant c_{7, \sigma}^{2}\left\|Z^{(m)}\right\|_{L_{2, \gamma}\left(\mathbb{R}_{+} ; \mathcal{H}_{\sigma}^{(m)}\right)}^{4}
$$

$$
\varepsilon^{2} v_{e}\left\|B^{(m)}\left(T^{-1}\left(Z^{(m)}\right), T^{-1}\left(Z^{(m)}\right)\right)\right\|_{L_{2, \gamma}\left(\mathbb{R}_{+} ; \mathcal{H}_{\sigma}^{(m)}\right)}^{2} \leqslant c_{8, \sigma}^{2}\left\|Z^{(m)}\right\|_{L_{2, \gamma}\left(\mathbb{R}_{+} ; \mathcal{H}_{\sigma}^{(m)}\right)}^{4}
$$

Остальные оценки доказываются аналогично.

7. Существование решения нелинейного уравнения. Решение нелинейного уравнения (9) найдем последовательностью итераций $X^{(j)}=\left(X_{k}^{(j)}, 1 \leqslant|k| \leqslant m\right)$ :

$$
\begin{gathered}
X_{k}^{(j)}=e^{-L_{e} t / \varepsilon} \mathcal{F}_{k}^{(m)}(t)-\varepsilon v_{e}^{1 / 2} \mathcal{H}_{k}^{(m)}(t)- \\
-\varepsilon v_{e}^{1 / 2}\left(\mathcal{U}_{k}^{(m)}\left(T^{-1}\left(X^{(j-1)}\right)\right)+B_{k}^{(m)}\left(T^{-1}\left(X^{(j-1)}\right), T^{-1}\left(X^{(j-1)}\right)\right)\right), \quad j \geqslant 1, \\
X_{k}^{(0)}=e^{-4 w_{e} t / \varepsilon} \mathcal{F}_{k}^{(m)}(t)-\varepsilon v_{e}^{1 / 2} \mathcal{H}_{k}^{(m)}(t) .
\end{gathered}
$$

Здесь

$$
\mathcal{F}_{k}^{(m)}(t)=F_{k, L}^{(m)}(t)+F_{k, B}^{(m)}(t), \quad \mathcal{H}_{k}^{(m)}(t)=H_{k, L}^{(m)}(t)+H_{k, B}^{(m)}(t) .
$$


Введем обозначения

$$
\widehat{\mathcal{F}^{(m)}}=e^{-L_{e} t / \varepsilon} \mathcal{F}_{k}^{(m)}(t)-\varepsilon v_{e}^{1 / 2} \mathcal{H}_{k}^{(m)}(t), \quad \widehat{\mathcal{F}^{(m)}}=\left(\widehat{\mathcal{F}_{k}^{(m)}},|k| \leqslant m, k \in \mathbb{Z}_{0}\right) .
$$

Сформулируем теоремы ограниченности и фундаментальности последовательности итераций. Отсюда будет следовать теорема существования решения нелинейного уравнения.

Теорема 3. Пусть $\sigma>2$ и существует такое $q \in(0,1)$, не зависящее от $m$, $\varepsilon$, что

$$
\frac{1}{1-q} c_{1, \sigma}\|\widehat{\mathcal{F}(m)}\|_{L_{2, \gamma}\left(\mathbb{R}_{+} ; \mathcal{H}_{\sigma}^{(m)}\right)}+\frac{1}{\sqrt{\varepsilon}} c_{4, \sigma}\left(\left\|\widehat{u}^{0}\right\|_{\mathcal{H}_{\sigma}^{(m)}}+\left\|\widehat{v}^{0}\right\|_{\mathcal{H}_{\sigma}^{(m)}}+\left\|\widehat{w}^{0}\right\|_{\mathcal{H}_{\sigma}^{(m)}}\right) \leqslant q, \quad q \in(0,1) .
$$

Тогда для любого $j \geqslant 1$ справедлива оченка

$$
\left\|X^{(j)}\right\|_{L_{2, \gamma}\left(\mathbb{R}_{+} ; \mathcal{H}_{\sigma}^{(m)}\right)} \leqslant \frac{1}{1-q}\|\widehat{\mathcal{F}(m)}\|_{L_{2, \gamma}\left(\mathbb{R}_{+} ; \mathcal{H}_{\sigma}^{(m)}\right)} .
$$

Теорема 4. Последовательность $X^{(j)}$ является фундаментальной в $L_{2, \gamma}\left(\mathbb{R}_{+} ; \mathcal{H}_{\sigma}^{(m)}\right)$, если

$$
\frac{2}{1-q} c_{1, \sigma}\|\widehat{\mathcal{F}(m)}\|_{L_{2, \gamma}\left(\mathbb{R}_{+} ; \mathcal{H}_{\sigma}^{(m)}\right)}+\frac{1}{\sqrt{\varepsilon}} c_{4, \sigma}\left(\left\|\widehat{u}^{0}\right\|_{\mathcal{H}_{\sigma}^{(m)}}+\left\|\widehat{v}^{0}\right\|_{\mathcal{H}_{\sigma}^{(m)}}+\left\|\widehat{w}^{0}\right\|_{\mathcal{H}_{\sigma}^{(m)}}\right) \leqslant q_{1}, \quad q_{1} \in(0,1) .
$$

Теорема 5. Пусть $\sigma>2$. Тогда существует единственное решение $Z^{(m)} \in L_{2, \gamma}\left(\mathbb{R}_{+} ; \mathcal{H}_{\sigma}^{(m)}\right)$ нелинейного уравнения (9), если

$$
\frac{2}{1-q} c_{1, \sigma}\|\widehat{\mathcal{F}(m)}\|_{L_{2, \gamma}\left(\mathbb{R}_{+} ; \mathcal{H}_{\sigma}^{(m)}\right)}+c_{4, \sigma} \frac{1}{\sqrt{\varepsilon}}\left(\left\|\widehat{u}^{0}\right\|_{\mathcal{H}_{\sigma}^{(m)}}+\left\|\widehat{v}^{0}\right\|_{\mathcal{H}_{\sigma}^{(m)}}+\left\|\widehat{w}^{0}\right\|_{\mathcal{H}_{\sigma}^{(m)}}\right) \leqslant q_{2}, \quad q_{2} \in(0,1),
$$

для которого выполняется неравенство

$$
\left\|Z^{(m)}\right\|_{L_{2, \gamma}\left(\mathbb{R}_{+} ; \mathcal{H}_{\sigma}^{(m)}\right)} \leqslant \frac{1}{1-q}\|\widehat{\mathcal{F}(m)}\|_{L_{2, \gamma}\left(\mathbb{R}_{+} ; \mathcal{H}_{\sigma}^{(m)}\right)} .
$$

Здесь $q$ определяется теоремой ограниченности последовательности итераций. Перепишем теорему существования решения через начальные данные.

Теорема 6. Пусть $\sigma>2$. Тогда существует единственное решение $Z^{(m)} \in L_{2, \gamma}\left(\mathbb{R}_{+} ; \mathcal{H}_{\sigma}^{(m)}\right)$ нелинейного уравнения (9), если

$$
\left(\left\|\widehat{u}^{0}\right\|_{\mathcal{H}_{\sigma}^{(m)}}+\left\|\widehat{v}^{0}\right\|_{\mathcal{H}_{\sigma}^{(m)}}+\left\|\widehat{w}^{0}\right\|_{\mathcal{H}_{\sigma}^{(m)}}\right) \leqslant \varepsilon^{3 / 4} q_{3}, \quad q_{3} \in(0,1),
$$

для которого выполняется неравенство

$$
\left\|Z^{(m)}\right\|_{L_{2, \gamma}\left(\mathbb{R}_{+} ; \mathcal{H}_{\sigma}^{(m)}\right)} \leqslant \frac{Q_{2, \sigma}}{\varepsilon}\left(\left\|\widehat{u}^{0}\right\|\left\|_{\mathcal{H}_{\sigma}^{(m)}}+\right\| \widehat{v}^{0}\left\|_{\mathcal{H}_{\sigma}^{(m)}}+\right\| \widehat{w}^{0} \|_{\mathcal{H}_{\sigma}^{(m)}}\right),
$$

где

$$
Q_{2, \sigma}=\frac{1}{1-q}\left(c_{2} \sqrt{\varepsilon}+c_{2, \sigma} \varepsilon^{1 / 4} q_{4}\right)
$$

8. Аппроксимационное решение возмущенной системы Годунова-Султангазина. Рассмотрим последовательность аппроксимационных решений

$$
\begin{gathered}
\widehat{u}^{(m)}(x, t)=u_{0}^{(m)}(t)+\sum_{\substack{k \in \mathbb{Z}_{0},|k| \leqslant m}} u_{k}^{(m)}(t) e^{i k x}, \quad \widehat{v}^{(m)}(x, t)=v_{0}^{(m)}(t)+\sum_{\substack{k \in \mathbb{Z}_{0},|k| \leqslant m}} v_{k}^{(m)}(t) e^{i k x}, \\
\widehat{w}^{(m)}(x, t)=w_{0}^{(m)}(t)+\sum_{\substack{k \in \mathbb{Z}_{0},|k| \leqslant m}} w_{k}^{(m)}(t) e^{i k x} .
\end{gathered}
$$

Теорема 7. Последовательность аппроксимационных решений фундаментальна по норме гильбертова пространства $W_{2, \gamma}^{1}\left(\mathbb{R}_{+} ; \mathcal{H}_{\sigma}\right)$. Более того, она сходится $\kappa$ слабому решению $\{\widehat{u}, \widehat{v}, \widehat{w}\}$ задачи Коши (3): 


$$
\begin{array}{r}
\int_{0}^{\infty} \int_{-\infty}^{\infty}\left(\widehat{u}\left(\partial_{t}+\partial_{x}\right)+\frac{1}{\varepsilon} w_{e}^{1 / 2}\left(2 v_{e}^{1 / 2} \widehat{v}-u_{e}^{1 / 2} \widehat{w}-w_{e}^{1 / 2} \widehat{u}\right)+\varepsilon w_{e}^{1 / 2}\left(\widehat{v}^{2}-\widehat{u} \widehat{w}\right)\right) \varphi(x, t) d x d t+ \\
+\left.\int_{-\infty}^{\infty} \widehat{u}^{0} \varphi(x, t)\right|_{t=0} d x=0
\end{array}
$$

$$
\begin{array}{r}
\int_{0}^{\infty} \int_{-\infty}^{\infty}\left(\widehat{v} \partial_{t}-\frac{2}{\varepsilon} v_{e}^{1 / 2}\left(2 v_{e}^{1 / 2} \widehat{v}-u_{e}^{1 / 2} \widehat{w}-w_{e}^{1 / 2} \widehat{u}\right)-2 \varepsilon v_{e}^{1 / 2}\left(\widehat{v}^{2}-\widehat{u} \widehat{w}\right)\right) \psi(x, t) d x d t+ \\
+\left.\int_{-\infty}^{\infty} \widehat{v}^{0} \psi(x, t)\right|_{t=0} d x=0
\end{array}
$$

$$
\begin{aligned}
& \int_{0}^{\infty} \int_{-\infty}^{\infty}\left(\widehat{w}\left(\partial_{t}-\partial_{x}\right)+\frac{1}{\varepsilon} u_{e}^{1 / 2}\left(2 v_{e}^{1 / 2} \widehat{v}-u_{e}^{1 / 2} \widehat{w}-w_{e}^{1 / 2} \widehat{u}\right)+\varepsilon u_{e}^{1 / 2}\left(\widehat{v}^{2}-\widehat{u} \widehat{w}\right)\right) \eta(x, t) d x d t+ \\
& +\left.\int_{-\infty}^{\infty} \widehat{w}^{0} \eta(x, t)\right|_{t=0} d x=0
\end{aligned}
$$

для любых тестовых функиий $\varphi, \psi, \eta \in C^{\infty}\left(\mathbb{R}_{+} ; C_{0}^{\infty}\left(R^{1}\right)\right)$ и существует такое $T$, что $\varphi(x, T)=$ $\psi(x, T)=\eta(x, T)=0$.

Доказательство. Докажем, что предел

$$
\widehat{u}(t, x)=u_{0}(t)+\sum_{k \in \mathbb{Z}_{0}} u_{k}(t) e^{i k x}, \quad \widehat{v}(t, x)=v_{0}(t)+\sum_{k \in \mathbb{Z}_{0}} v_{k}(t) e^{i k x}, \quad \widehat{w}(t, x)=w_{0}(t)+\sum_{k \in \mathbb{Z}_{0}} w_{k}(t) e^{i k x}
$$

в $L_{2, \gamma}\left(\mathbb{R}_{+} ; \mathcal{H}_{\sigma}\right)$ аппроксимационных решений $(25)$ есть слабое решение задачи Коши (3). Докажем справедливость первого интегрального тождества (26). Для этого добавим и вычтем интегральное тождество для $\widehat{u}^{(m)}$ в левой части (26). Имеем

$$
\begin{aligned}
& \int_{0}^{\infty} \int_{-\infty}^{\infty}\left(-\widehat{u}\left(\partial_{t}+\partial_{x}\right) \varphi(x, t)-\frac{1}{\varepsilon} w_{e}^{1 / 2}\left(2 v_{e}^{1 / 2} \widehat{v}-u_{e}^{1 / 2} \widehat{w}-w_{e}^{1 / 2} \widehat{u}\right) \varphi(x, t)-\right. \\
& \left.-\varepsilon w_{e}^{1 / 2}\left(\widehat{v}^{2}-\widehat{u} \widehat{w}\right) \varphi(x, t)\right) d x d t-\left.\int_{-\infty}^{\infty} \widehat{u}^{0} \varphi(x, t)\right|_{t=0} d x= \\
& =\int_{0}^{\infty} \int_{-\infty}^{\infty}\left(-\widehat{u}^{(m)}\left(\partial_{t}+\partial_{x}\right) \varphi(x, t)-\frac{1}{\varepsilon} w_{e}^{1 / 2}\left(2 v_{e}^{1 / 2} \widehat{v}^{(m)}-u_{e}^{1 / 2} \widehat{w}^{(m)}-w_{e}^{1 / 2} \widehat{u}^{(m)}\right) \varphi(x, t)-\right. \\
& \left.-\varepsilon w_{e}^{1 / 2}\left(\left(\widehat{v}^{(m)}\right)^{2}-\widehat{u}^{(m)} \widehat{w}^{(m)}\right) \varphi(x, t)\right) d x d t-\left.\left.\int_{-\infty}^{\infty} \widehat{u}^{(m)}\right|_{t=0} \varphi(x, t)\right|_{t=0} d x- \\
& -\int_{0}^{\infty} \int_{-\infty}^{\infty}\left(\left(\widehat{u}-\widehat{u}^{(m)}\right) \partial_{t} \varphi(x, t)+\left(\widehat{u}-\widehat{u}^{(m)}\right) \partial_{x} \varphi(x, t)\right) d x d t+ \\
& +\int_{0}^{\infty} \int_{-\infty}^{\infty}\left(-\frac{1}{\varepsilon} w_{e}^{1 / 2}\left(2 v_{e}^{1 / 2}\left(\widehat{v}-\widehat{v}^{(m)}\right)-u_{e}^{1 / 2}\left(\widehat{w}-\widehat{w}^{(m)}\right)-w_{e}^{1 / 2}\left(\widehat{u}-\widehat{u}^{(m)}\right)\right)-\right.
\end{aligned}
$$




$$
\begin{aligned}
-\varepsilon w_{e}^{1 / 2}\left((\widehat{v})^{2}-\left(\widehat{v}^{(m)}\right)^{2}-\right. & \left.\left.\left(\widehat{u} \widehat{w}-\widehat{u}^{(m)} \widehat{w}^{(m)}\right)\right)\right) \varphi(x, t) d x d t- \\
& -\int_{-\infty}^{\infty}\left(\widehat{u}(x, 0)-\widehat{u}^{(m)}(x, 0)\right) \varphi(x, 0) d x=I_{1}+I_{2}+I_{3},
\end{aligned}
$$

где

$$
\begin{gathered}
I_{1}=\int_{0}^{\infty} \int_{-\infty}^{\infty}\left(-\widehat{u}^{(m)}\left(\partial_{t}+\partial_{x}\right) \varphi(x, t)-\frac{1}{\varepsilon} w_{e}^{1 / 2}\left(2 v_{e}^{1 / 2} \widehat{v}^{(m)}-u_{e}^{1 / 2} \widehat{w}^{(m)}-w_{e}^{1 / 2} \widehat{u}^{(m)}\right) \varphi(x, t)-\right. \\
\left.\quad-\varepsilon w_{e}^{1 / 2}\left(\left(\widehat{v}^{(m)}\right)^{2}-\widehat{u}^{(m)} \widehat{w}^{(m)}\right) \varphi(x, t)\right) d x d t-\left.\left.\int_{-\infty}^{\infty} \widehat{u}^{(m)}\right|_{t=0} \varphi(x, t)\right|_{t=0} d x \\
-\int_{0-\infty}^{\infty} \int_{-\infty}^{\infty}\left(\left(\widehat{u}-\widehat{u}^{(m)}\right) \partial_{t} \varphi(x, t)+\left(\widehat{u}-\widehat{u}^{(m)}\right) \partial_{x} \varphi(x, t)\right) d x d t+ \\
+\int_{0}^{\infty} \int_{-\infty}^{\infty}\left(-\frac{1}{\varepsilon} w_{e}^{1 / 2}\left(2 v_{e}^{1 / 2}\left(\widehat{v}-\widehat{v}^{(m)}\right)-u_{e}^{1 / 2}\left(\widehat{w}-\widehat{w}^{(m)}\right)-w_{e}^{1 / 2}\left(\widehat{u}-\widehat{u}^{(m)}\right)\right)-\right. \\
\left.-\varepsilon w_{e}^{1 / 2}\left((\widehat{v})^{2}-\left(\widehat{v}^{(m)}\right)^{2}-\left(\widehat{u} \widehat{w}-\widehat{u}^{(m)} \widehat{w}^{(m)}\right)\right)\right) \varphi(x, t) d x d t- \\
-\int_{-\infty}^{\infty}\left(\widehat{u}^{(x, 0)}-\widehat{u}^{(m)}(x, 0)\right) \varphi(x, 0) d x=I_{2}+I_{3},
\end{gathered}
$$

$$
\begin{aligned}
& I_{2}=\int_{0}^{\infty} \int_{-\infty}^{\infty}\left(-\left(u_{0}(t)+\sum_{\substack{k \in \mathbb{Z}_{0},|k| \leqslant m}} u_{k}(t) e^{i k x}-u_{0}^{(m)}(t)-\sum_{\substack{k \in \mathbb{Z}_{0},|k| \leqslant m}} u_{k}^{(m)}(t) e^{i k x}\right)\left(\partial_{t}+\partial_{x}\right) \varphi(x, t)-\right. \\
& -\frac{1}{\varepsilon} w_{e}^{1 / 2}\left(2 v_{e}^{1 / 2}\left(v_{0}(t)+\sum_{\substack{k \in \mathbb{Z}_{0},|k| \leqslant m}} v_{k}(t) e^{i k x}-v_{0}^{(m)}(t)-\sum_{\substack{k \in \mathbb{Z}_{0},|k| \leqslant m}} v_{k}^{(m)}(t) e^{i k x}\right)-\right. \\
& -u_{e}^{1 / 2}\left(w_{0}(t)+\sum_{\substack{k \in \mathbb{Z}_{0},|k| \leqslant m}} w_{k}(t) e^{i k x}-w_{0}^{(m)}(t)-\sum_{\substack{k \in \mathbb{Z}_{0},|k| \leqslant m}} w_{k}^{(m)}(t) e^{i k x}\right)- \\
& \left.-w_{e}^{1 / 2}\left(u_{0}(t)+\sum_{\substack{k \in \mathbb{Z}_{0},|k| \leqslant m}} u_{k}(t) e^{i k x}-u_{0}^{(m)}(t)-\sum_{\substack{k \in \mathbb{Z}_{0},|k| \leqslant m}} u_{k}^{(m)}(t) e^{i k x}\right)\right) \varphi(x, t)- \\
& -\varepsilon w_{e}^{1 / 2}\left(\left(v_{0}(t)+\sum_{\substack{k \in \mathbb{Z}_{0},|k| \leqslant m}} v_{k}(t) e^{i k x}\right)^{2}-\left(v_{0}^{(m)}(t)+\sum_{\substack{k \in \mathbb{Z}_{0},|k| \leqslant m}} v_{k}^{(m)}(t) e^{i k x}\right)^{2}-\right. \\
& -\left(\left(u_{0}(t)+\sum_{\substack{k \in \mathbb{Z}_{0},|k| \leqslant m}} u_{k}(t) e^{i k x}\right)\left(w_{0}(t)+\sum_{\substack{k \in \mathbb{Z}_{0},|k| \leqslant m}} w_{k}(t) e^{i k x}\right)-\right.
\end{aligned}
$$




$$
\left.\left.-\left(u_{0}^{(m)}(t)+\sum_{\substack{k \in \mathbb{Z}_{0},|k| \leqslant m}} u_{k}^{(m)}(t) e^{i k x}\right)\left(w_{0}^{(m)}(t)+\sum_{\substack{k \in \mathbb{Z}_{0},|k| \leqslant m}} w_{k}^{(m)}(t) e^{i k x}\right)\right) \varphi(x, t)\right) d x d t .
$$

Здесь мы воспользовались тем, что $\left.u_{k}(t)\right|_{t=0}=\left.u_{k}^{(m)}(t)\right|_{t=0}$ для любого $|k| \leqslant m, k \in \mathbb{Z}$, т.е.

$$
-\left.\left.\int_{-\infty}^{\infty}\left(u_{0}(t)+\sum_{\substack{k \in \mathbb{Z}_{0},|k| \leqslant m}} u_{k}(t) e^{i k x}-u_{0}^{(m)}(t)-\sum_{\substack{k \in \mathbb{Z}_{0},|k| \leqslant m}} u_{k}^{(m)}(t) e^{i k x}\right)\right|_{t=0} \varphi(x, t)\right|_{t=0} d x=0 .
$$

В то же время

$$
\begin{aligned}
& I_{3}= \int_{0}^{\infty} \int_{-\infty}^{\infty}\left[-\sum_{\substack{k \in \mathbb{Z}_{0},|k|>m}} u_{k}(t) e^{i k x}\left(\partial_{t}+\partial_{x}\right)-\right. \\
&-\frac{1}{\varepsilon} w_{e}^{1 / 2}\left(2 v_{e}^{1 / 2} \sum_{\substack{k \in \mathbb{Z}_{0},|k|>m}} v_{k}(t) e^{i k x}-u_{e}^{1 / 2} \sum_{\substack{k \in \mathbb{Z}_{0},|k|>m}} w_{k}(t) e^{i k x}-w_{e}^{1 / 2} \sum_{\substack{k \in \mathbb{Z}_{0},|k|>m}} u_{k}(t) e^{i k x}\right)- \\
&-\varepsilon w_{e}^{1 / 2}\left(\left(\sum_{\substack{k \in \mathbb{Z}_{0},|k|>m}} v_{k}(t) e^{i k x}\right)^{2}-\left(\sum_{\substack{k \in \mathbb{Z}_{0},|k|>m}} u_{k}(t) e^{i k x}\right)\left(\sum_{\substack{k \in \mathbb{Z}_{0},|k|>m}} w_{k}(t) e^{i k x}\right)\right)- \\
&-\varepsilon w_{e}^{1 / 2}\left(2\left(\sum_{\substack{k \in \mathbb{Z}_{0},|k|>m}} v_{k}(t) e^{i k x}\right)\left(v_{0}(t)+\sum_{\substack{k \in \mathbb{Z}_{0},|k| \leqslant m}} v_{k}(t) e^{i k x}\right)-\left(\sum_{\substack{k \in \mathbb{Z}_{0},|k|>m}} u_{k}(t) e^{i k x}\right)\left(w_{0}(t)+\sum_{\substack{k \in \mathbb{Z}_{0},|k| \leqslant m}} w_{k}(t) e^{i k x}\right)-\right. \\
&\left.\left.-\left(u_{0}(t)+\sum_{\substack{k \in \mathbb{Z}_{0},|k| \leqslant m}}^{\infty} u_{k}(t) e^{i k x}\right)\left(\sum_{\substack{k \in \mathbb{Z}_{0},|k|>m}} w_{k}(t) e^{i k x}\right)\right)\right] \varphi(x, t) d x d t-\left.\int_{-\infty}^{\substack{k \in \mathbb{Z}_{0},|k|>m}} u_{k}(0) e^{i k x} \varphi(x, t)\right|_{t=0} d x .
\end{aligned}
$$

Далее, интегрируя по частям, получим

$$
\begin{array}{r}
I_{1}=\int_{0}^{\infty} \int_{-\infty}^{\infty}\left(\left(\partial_{t}+\partial_{x}\right) \widehat{u}^{(m)}-\frac{1}{\varepsilon} w_{e}^{1 / 2}\left(2 v_{e}^{1 / 2} \widehat{v}^{(m)}-u_{e}^{1 / 2} \widehat{w}^{(m)}-w_{e}^{1 / 2} \widehat{u}^{(m)}\right)-\right. \\
\left.-\varepsilon w_{e}^{1 / 2}\left(\left(\widehat{v}^{(m)}\right)^{2}-\widehat{u}^{(m)} \widehat{w}^{(m)}\right)\right) \varphi(x, t) d x d t .
\end{array}
$$

Обозначим

$$
\begin{aligned}
& \left|I_{0}\right|=\int_{0}^{\infty} \int_{-\infty}^{\infty}\left|\left(\widehat{u}-\widehat{u}^{(m)}\right) \partial_{t} \varphi(x, t)\right| d x d t \leqslant \\
& \leqslant \int_{0}^{\infty} \int_{-\infty}^{\infty}\left|\sum_{\substack{k \in \mathbb{Z}_{0},|k| \leqslant m}}\left(u_{k}-u_{k}^{(m)}\right) e^{i k x} \partial_{t} \varphi(x, t)\right| d x d t+\int_{0}^{\infty} \int_{-\infty}^{\infty}\left|\sum_{|k|>m} u_{k} e^{i k x} \partial_{t} \varphi(x, t)\right| d x d t=\left|J_{1}\right|+\left|J_{2}\right|
\end{aligned}
$$

Применяем неравенство Гельдера и пользуемся тем, что носитель по переменной $x$ изменяется от $[-R ; R]$, получим 


$$
\begin{gathered}
\left|J_{1}\right| \leqslant\left(\int_{0}^{\infty} \int_{-R}^{R}\left|\sum_{\substack{k \in \mathbb{Z}_{0},|k| \leqslant m}} \frac{|k|^{2 \sigma}}{|k|^{2 \sigma}}\left(u_{k}-u_{k}^{(m)}\right) e^{i k x}\right|^{2} d x d t\right)^{1 / 2}\left(\int_{0}^{\infty} \int_{-\infty}^{\infty}\left|\partial_{t} \varphi(x, t)\right|^{2} d x d t\right)^{1 / 2} \leqslant \\
\leqslant c_{1}\left(\int_{0}^{\infty} e^{2 \gamma t} \sup _{\substack{k \in \mathbb{Z}_{0},|k| \leqslant m}}|k|^{2 \sigma}\left|\left(u_{k}-u_{k}^{(m)}\right)\right|^{2} d t\right)^{1 / 2}\left(\int_{0}^{\infty} \int_{-\infty}^{\infty}\left|\partial_{t} \varphi(x, t)\right|^{2} d x d t\right)^{1 / 2} \leqslant \\
\leqslant c_{1}\left\|\widehat{u}-\widehat{u}^{(m)}\right\|_{L_{2, \gamma}\left(\mathbb{R}_{+} ; \mathcal{H}_{\sigma}^{(m)}\right)}\left(\int_{0}^{\infty} \int_{-\infty}^{\infty}\left|\partial_{t} \varphi(x, t)\right|^{2} d x d t\right)^{1 / 2} \rightarrow 0, \quad m \rightarrow \infty
\end{gathered}
$$

в силу фундаментальности $\widehat{u}^{(m)}$. Далее,

$$
\begin{gathered}
\left|J_{2}\right|^{2}=\left(\int_{0}^{\infty} \int_{-\infty}^{\infty}\left|\sum_{|k|>m} u_{k} e^{i k x} \partial_{t} \varphi(x, t)\right| d x d t\right)^{2} \\
\leqslant \int_{0}^{\infty} \int_{-R}^{R}\left|\sum_{|k|>m} u_{k} e^{i k x}\right|^{2} d x d t \int_{0}^{\infty} \int_{-\infty}^{\infty}\left|\partial_{t} \varphi(x, t)\right|^{2} d x d t \leqslant \\
\leqslant c_{2}\|\widehat{u}\|_{L_{2, \gamma}\left(\mathbb{R}_{+} ; \mathcal{H}_{\sigma}\right)}^{2} \sum_{|k|>m} \frac{1}{|k|^{2 \sigma}} \int_{0}^{\infty} \int_{-\infty}^{\infty}\left|\partial_{t} \varphi(x, t)\right|^{2} d x d t .
\end{gathered}
$$

Для оценки суммы воспользуемся следующим неравенством (доказательство см. в [5])

$$
\sum_{|k|>m} \frac{1}{|k|^{2 \sigma}} \leqslant c_{\sigma}^{\text {sum }} \frac{1}{m^{2 \sigma-1}}
$$

Тогда

$$
\left|J_{2}\right|^{2} \leqslant\|\widehat{u}\|_{L_{2, \gamma}\left(\mathbb{R}_{+} ; \mathcal{H}_{\sigma}\right)}^{2} c_{\sigma}^{\text {sum }} \frac{1}{m^{2 \sigma-1}} \int_{0}^{\infty} \int_{-\infty}^{\infty}\left|\partial_{t} \varphi(x, t)\right|^{2} d x d t \rightarrow 0, \quad m \rightarrow \infty .
$$

Аналогично доказывается, что при $m \rightarrow \infty$

$$
\begin{gathered}
\left|U_{1}\right|=\int_{0}^{\infty} \int_{-\infty}^{\infty}\left|\left(\widehat{u}-\widehat{u}^{(m)}\right) \partial_{x} \varphi(x, t)\right| d x d t \rightarrow 0 \\
\left|U_{2}\right|=\int_{0}^{\infty} \int_{-\infty}^{\infty}\left|\frac{1}{\varepsilon} w_{e}^{1 / 2}\left(2 v_{e}^{1 / 2}\left(\widehat{v}-\widehat{v}^{(m)}\right)-u_{e}^{1 / 2}\left(\widehat{w}-\widehat{w}^{(m)}\right)-w_{e}^{1 / 2}\left(\widehat{u}-\widehat{u}^{(m)}\right)\right) \varphi(x, t)\right| d x d t \rightarrow 0 .
\end{gathered}
$$

Отсюда следует, что

$$
\begin{aligned}
\int_{0}^{\infty} \int_{-\infty}^{\infty}\left(-\left(\widehat{u}-\widehat{u}^{(m)}\right) \partial_{t}-\left(\widehat{u}-\widehat{u}^{(m)}\right) \partial_{x}-\frac{1}{\varepsilon} w_{e}^{1 / 2}\left(2 v_{e}^{1 / 2}\left(\widehat{v}-\widehat{v}^{(m)}\right)-\right.\right. & \\
& \left.\left.-u_{e}^{1 / 2}\left(\widehat{w}-\widehat{w}^{(m)}\right)-w_{e}^{1 / 2}\left(\widehat{u}-\widehat{u}^{(m)}\right)\right)\right) \varphi(x, t) d x d t \rightarrow 0, \quad m \rightarrow \infty
\end{aligned}
$$

Теперь покажем, что

$$
\varepsilon w_{e}^{1 / 2} \int_{0}^{\infty} \int_{-\infty}^{\infty}\left((\widehat{v})^{2}-\left(\widehat{v}^{(m)}\right)^{2}-\left(\widehat{u} \widehat{w}-\widehat{u}^{(m)} \widehat{w}^{(m)}\right)\right) \varphi(x, t) d x d t \rightarrow 0, \quad m \rightarrow \infty .
$$


Рассмотрим разность

$$
\begin{aligned}
&\left|U_{3}\right|=\int_{0}^{\infty} \int_{-\infty}^{\infty}\left|\left((\widehat{v})^{2}-\left(\widehat{v}^{(m)}\right)^{2}\right) \varphi(x, t)\right| d x d t= \\
&=\int_{0}^{\infty} \int_{-\infty}^{\infty}\left|\left(\sum_{\substack{k_{1}+k_{2}=k, k_{1}, k_{2} \in \mathbb{Z}_{0}}} v_{k_{1}} v_{k_{2}}-\sum_{\substack{k_{1}+k_{2}=k,\left|k_{1}\right|,\left|k_{2}\right| \leqslant m, k_{1}, k_{2} \in \mathbb{Z}_{0}}} v_{k_{1}}^{(m)} v_{k_{2}}^{(m)}\right) e^{i k x} \varphi(x, t)\right| d x d t .
\end{aligned}
$$

Заметим, что

$$
v_{k_{1}} v_{k_{2}}-v_{k_{1}}^{(m)} v_{k_{2}}^{(m)}=\left(v_{k_{1}}-v_{k_{1}}^{(m)}\right) v_{k_{2}}+\left(v_{k_{2}}-v_{k_{2}}^{(m)}\right) v_{k_{1}}^{(m)} .
$$

Тогда

$$
\begin{aligned}
\left|U_{3}\right| \leqslant \int_{0}^{\infty} \int_{-\infty}^{\infty} \mid \sum_{\substack{k_{1}+k_{2}=k,\left|k_{1}\right|,\left|k_{2}\right| \leqslant m, k_{1}, k_{2} \in \mathbb{Z}_{0}}}\left(\left(v_{k_{1}}-v_{k_{1}}^{(m)}\right) v_{k_{2}}\right. & \left.+\left(v_{k_{2}}-v_{k_{2}}^{(m)}\right) v_{k_{1}}^{(m)}\right) e^{i k x} \varphi(x, t) \mid d x d t+ \\
& +\int_{0}^{\infty} \int_{-\infty}^{\infty}\left|\sum_{\substack{k_{1}+k_{2}=k,\left|k_{1}\right|,\left|, k_{2}\right|>m, k_{1}, k_{2} \in \mathbb{Z}_{0}}} v_{k_{1}} v_{k_{2}} e^{i k x} \varphi(x, t)\right| d x d t=\left|U_{4}\right|+\left|U_{5}\right| .
\end{aligned}
$$

Далее,

$$
\begin{gathered}
\left|U_{4}\right|=\int_{0}^{\infty} \int_{-\infty}^{\infty}\left|\sum_{\substack{k_{1}+k_{2}=k,\left|k_{1}\right|,\left|k_{2}\right| \leqslant m, k_{1}, k_{2} \in \mathbb{Z}_{0}}}\left(\left(v_{k_{1}}-v_{k_{1}}^{(m)}\right) v_{k_{2}}+\left(v_{k_{2}}-v_{k_{2}}^{(m)}\right) v_{k_{1}}^{(m)}\right) e^{i k x} \varphi(x, t)\right| d x d t \leqslant \\
\leqslant \int_{0}^{\infty} \int_{-\infty}^{\infty} \sum_{\substack{k_{1}+k_{2}=k,\left|k_{1}\right|,\left|k_{2}\right| \leqslant m, k_{1}, k_{2} \in \mathbb{Z}_{0}}} \frac{\left|k_{1}\right|^{\sigma}}{\left|k_{1}\right|^{\sigma}} \frac{\left|k_{2}\right|^{\sigma}}{\left|k_{2}\right|^{\sigma}}\left|\left(v_{k_{1}}-v_{k_{1}}^{(m)}\right) v_{k_{2}} e^{i k x} \varphi(x, t)\right| d x d t+ \\
+\int_{0}^{\infty} \int_{-\infty}^{\infty} \sum_{\substack{k_{1}+k_{2}=k, k_{1}|,| k_{2} \mid \leqslant m, k_{1}, k_{2} \in \mathbb{Z}_{0}}}^{\infty} \frac{\left|k_{1}\right|^{\sigma}}{\left|k_{1}\right|^{\sigma}} \frac{\left|k_{2}\right|^{\sigma}}{\left|k_{2}\right|^{\sigma}}\left|\left(v_{k_{2}}-v_{k_{2}}^{(m)}\right) v_{k_{1}}^{(m)} e^{i k x} \varphi(x, t)\right| d x d t=\left|I_{5}\right|+\left|I_{6}\right| .
\end{gathered}
$$

Рассмотрим $I_{5}$ :

$$
\begin{aligned}
& \left|I_{5}\right|^{2}=\left(\int_{0}^{\infty} \int_{-\infty}^{\infty} \sum_{\substack{k_{1}+k_{2}=k,\left|k_{1}\right|,\left|k_{2}\right| \leqslant m, k_{1}, k_{2} \in \mathbb{Z}_{0}}} \frac{\left|k_{1}\right|^{\sigma}}{\left|k_{1}\right|^{\sigma}} \frac{\left|k_{2}\right|^{\sigma}}{\left|k_{2}\right|^{\sigma}}\left|\left(v_{k_{1}}-v_{k_{1}}^{(m)}\right) v_{k_{2}} e^{i k x} \varphi(x, t)\right| d x d t\right)^{2} \leqslant \\
& \leqslant 2 \sup _{t \in \mathbb{R}_{+}} \sup _{\substack{k_{2} \in \mathbb{Z}_{0},\left|k_{2}\right| \leqslant m}}\left|k_{2}\right|^{2 \sigma}\left|v_{k_{2}}\right|^{2}\left(\int_{0}^{\infty} \int_{-\infty}^{\infty} \sup _{\substack{k_{1} \in \mathbb{Z}_{0},\left|k_{1}\right| \leqslant m}}^{\infty}\left|k_{1}\right|^{\sigma}\left|\left(v_{k_{1}}-v_{k_{1}}^{(m)}\right) \varphi(x, t)\right| d x d t\right)^{2}\left(\sum_{\substack{\left|k_{1}\right|,\left|k_{2}\right| \leqslant m, k_{1}, k_{2} \in \mathbb{Z}_{0},}} \frac{1}{\left|k_{1}\right|^{\sigma}\left|k_{2}\right|^{\sigma}}\right)^{2} .
\end{aligned}
$$

Оценим множители по отдельности. Во-первых, 


$$
\begin{aligned}
& L=\sup _{t \in \mathbb{R}_{+}} \sup _{\substack{k_{2} \in \mathbb{Z}_{0},\left|k_{2}\right| \leqslant m}}\left|k_{2}\right|^{2 \sigma}\left|v_{k_{2}}(t)\right|^{2}=\left.\left.\sup _{t \in \mathbb{R}_{+}} \sup _{\substack{k_{2} \in \mathbb{Z}_{0},\left|k_{2}\right| \leqslant m}} \int_{0}^{t} \frac{d}{d s}|| k_{2}\right|^{\sigma} v_{k_{2}}(s)\right|^{2} d s \leqslant \\
& \leqslant\left. 2 \sup _{t \in \mathbb{R}_{+}} \sup _{\substack{k_{2} \in \mathbb{Z}_{0},\left|k_{2}\right| \leqslant m}} \int_{0}^{t}|| k_{2}\right|^{\sigma} v_{k_{2}}|| \frac{d}{d s}\left|k_{2}\right|^{\sigma} v_{k_{2}} \mid d s
\end{aligned}
$$

Используя неравенство Гельдера, получим

$$
L \leqslant 2\left(\left.\left.\int_{0}^{\infty} \sup _{\substack{k_{2} \in \mathbb{Z}_{0},\left|k_{2}\right| \leqslant m}}|| k_{2}\right|^{\sigma} v_{k_{2}}\right|^{2} d s\right)^{1 / 2}\left(\left.\left.\int_{\substack{0 \\ 0}}^{\infty} \sup _{\substack{k_{2} \in \mathbb{Z}_{0},\left|k_{2}\right| \leqslant m}}\left|\frac{d}{d s}\right| k_{1}\right|^{\sigma} v_{k_{2}}\right|^{2} d s\right)^{1 / 2} .
$$

Заметим, что $1 \leqslant e^{2 \gamma s}$ для всех $s \geqslant 0$. Значит, можно переписать неравенство в виде

$$
\begin{aligned}
L \leqslant 2\left(\left.\left.\int_{0}^{\infty} e^{2 \gamma s} \sup _{\substack{k_{2} \in \mathbb{Z}_{0},\left|k_{2}\right| \leqslant m}}|| k_{2}\right|^{\sigma} v_{k_{2}}\right|^{2} d s\right)^{1 / 2}\left(\left.\left.\int_{0}^{\infty} e^{2 \gamma s} \sup _{\substack{k_{2} \in \mathbb{Z}_{0},\left|k_{2}\right| \leqslant m}}\left|\frac{d}{d s}\right| k_{2}\right|^{\sigma} v_{k_{2}}\right|^{2} d s\right)^{1 / 2}= & =2\|\widehat{v}\|_{L_{2, \gamma}\left(\mathbb{R}_{+} ; \mathcal{H}_{\sigma}^{(m)}\right)}\left\|\frac{d}{d t} \widehat{v}\right\|_{L_{2, \gamma}\left(\mathbb{R}_{+} ; \mathcal{H}_{\sigma}^{(m)}\right)} .
\end{aligned}
$$

Для второго множителя имеем

$$
\begin{aligned}
\left(\int_{0}^{\infty} \int_{-\infty}^{\infty} \sup _{\substack{k_{1} \in \mathbb{Z}_{0},\left|k_{1}\right| \leqslant m}}\left|k_{1}\right|^{\sigma}\left|\left(v_{k_{1}}-v_{k_{1}}^{(m)}\right) \varphi(x, t)\right| d x d t\right)^{2} & \leqslant \\
& \leqslant \int_{0}^{\infty} \int_{-R}^{R} e^{2 \gamma t} \sup _{\substack{k_{1} \in \mathbb{Z}_{0},\left|k_{1}\right| \leqslant m}}\left|k_{1}\right|^{\sigma}\left|v_{k_{1}}-v_{k_{1}}^{(m)}\right|^{2} d x d t \int_{0}^{\infty} \int_{-\infty}^{\infty}|\varphi(x, t)|^{2} d x d t= \\
& =c_{2}\left\|\widehat{v}-\widehat{v}^{(m)}\right\|_{L_{2, \gamma}\left(\mathbb{R}_{+} ; \mathcal{H}_{\sigma}^{(m)}\right)}^{2} \int_{0}^{\infty} \int_{-\infty}^{\infty}|\varphi(x, t)|^{2} d x d t .
\end{aligned}
$$

Отметим, что

$$
\sum_{\substack{\left|k_{1}\right|,\left|k_{2}\right| \leqslant m, k_{1}, k_{2} \in \mathbb{Z}_{0}}} \frac{1}{\left|k_{1}\right|^{\sigma}\left|k_{2}\right|^{\sigma}} \leqslant \sum_{k_{1}=1}^{\infty} \frac{1}{\left|k_{1}\right|^{\sigma}}+\sum_{k_{2}=1}^{\infty} \frac{1}{\left|k_{2}\right|^{\sigma}}
$$

Данные ряды сходятся при $\sigma>1$. Получаем

$$
\begin{aligned}
\left|I_{5}\right|^{2} \leqslant c_{3}\|\widehat{v}\|_{L_{2, \gamma}\left(\mathbb{R}_{+} ; \mathcal{H}_{\sigma}^{(m)}\right)}\left\|\frac{d}{d t} \widehat{v}\right\|_{L_{2, \gamma}\left(\mathbb{R}_{+} ; \mathcal{H}_{\sigma}^{(m)}\right)} & \times \\
& \times\left\|\widehat{v}-\widehat{v}^{(m)}\right\|_{L_{2, \gamma}\left(\mathbb{R}_{+} ; \mathcal{H}_{\sigma}^{(m)}\right)}^{2} \int_{0}^{\infty} \int_{-\infty}^{\infty}|\varphi(x, t)|^{2} d x d t \rightarrow 0, \quad m \rightarrow \infty
\end{aligned}
$$

в силу того, что выражения $\|\widehat{v}\|_{L_{2, \gamma}\left(\mathbb{R}_{+} ; \mathcal{H}_{\sigma}^{(m)}\right)},\|d \widehat{v} d t\|_{L_{2, \gamma}\left(\mathbb{R}_{+} ; \mathcal{H}_{\sigma}^{(m)}\right)}$ ограниченны $\left(\widehat{v}^{(m)}\right.$ фундаментальна и ограниченна в $\left.L_{2, \gamma}\left(\mathbb{R}_{+} ; \mathcal{H}_{\sigma}^{(m)}\right)\right)$, а $\left\|\widehat{v}-\widehat{v}^{(m)}\right\|_{L_{2, \gamma}\left(\mathbb{R}_{+} ; \mathcal{H}_{\sigma}^{(m)}\right)}^{2} \rightarrow 0$. Аналогично приходим к 
оценке для $I_{6}$ :

$$
\begin{aligned}
\left|I_{6}\right|^{2} \leqslant c_{4}\left\|\widehat{v}^{(m)}\right\|_{L_{2, \gamma}\left(\mathbb{R}_{+} ; \mathcal{H}_{\sigma}^{(m)}\right)}\left\|\frac{d}{d t} \widehat{v}^{(m)}\right\|_{L_{2, \gamma}\left(\mathbb{R}_{+} ; \mathcal{H}_{\sigma}^{(m)}\right)} \times \\
\quad \times\left\|\widehat{v}-\widehat{v}^{(m)}\right\|_{L_{2, \gamma}\left(\mathbb{R}_{+} ; \mathcal{H}_{\sigma}^{(m)}\right)}^{2} \int_{0}^{\infty} \int_{-\infty}^{\infty}|\varphi(x, t)|^{2} d x d t \rightarrow 0, \quad m \rightarrow \infty .
\end{aligned}
$$

Суммируя $\left|I_{5}\right|$ и $\left|I_{6}\right|$, находим

$$
\begin{aligned}
& \left|U_{3}\right|^{2} \leqslant c_{3}\|\widehat{v}\|_{L_{2, \gamma}\left(\mathbb{R}_{+} ; \mathcal{H}_{\sigma}^{(m)}\right)}\left\|\frac{d}{d t} \widehat{v}\right\|_{L_{2, \gamma}\left(\mathbb{R}_{+} ; \mathcal{H}_{\sigma}^{(m)}\right)}\left\|\widehat{v}-\widehat{v}^{(m)}\right\|_{L_{2, \gamma}\left(\mathbb{R}_{+} ; \mathcal{H}_{\sigma}^{(m)}\right)}^{2} \int_{0}^{\infty} \int_{-\infty}^{\infty}|\varphi(x, t)|^{2} d x d t+ \\
& \quad+c_{4}\left\|\widehat{v}^{(m)}\right\|_{L_{2, \gamma}\left(\mathbb{R}_{+} ; \mathcal{H}_{\sigma}^{(m)}\right)}\left\|\frac{d}{d t} \widehat{v}^{(m)}\right\|_{L_{2, \gamma}\left(\mathbb{R}_{+} ; \mathcal{H}_{\sigma}^{(m)}\right)}\left\|\widehat{v}-\widehat{v}^{(m)}\right\|_{L_{2, \gamma}\left(\mathbb{R}_{+} ; \mathcal{H}_{\sigma}^{(m)}\right)}^{2} \int_{0}^{\infty} \int_{-\infty}^{\infty}|\varphi(x, t)|^{2} d x d t \rightarrow 0
\end{aligned}
$$

при $m \rightarrow \infty$. Остаются слагаемые для $|k| \geqslant m+1$ :

$$
U_{6}=\int_{0}^{\infty} \int_{-\infty}^{\infty}\left|\sum_{\substack{k_{1}+k_{2}=k,|k|>m, k_{1}, k_{2} \in \mathbb{Z}_{0}}} v_{k_{1}} v_{k_{2}} e^{i k x} \varphi(x, t)\right| d x d t
$$

Заметим, что имеет место вложение множеств

$$
\begin{aligned}
& \left\{\left|k_{1}+k_{2}\right|>m, k_{1}, k_{2} \in \mathbb{Z}_{0}\right\} \subset\left\{\left\{\left|k_{1}\right| \leqslant \frac{m}{2},\left|k_{2}\right|>\frac{m}{2}, k_{1}, k_{2} \in \mathbb{Z}_{0}\right\} \cup\right. \\
& \left.\cup\left\{\left|k_{2}\right| \leqslant \frac{m}{2},\left|k_{1}\right|>\frac{m}{2}, k_{1}, k_{2} \in \mathbb{Z}_{0}\right\} \cup\left\{\left|k_{1}\right|>\frac{m}{2},\left|k_{2}\right|>\frac{m}{2}, k_{1}, k_{2} \in \mathbb{Z}_{0}\right\}\right\} .
\end{aligned}
$$

Воспользуемся неравенством

$$
(a+b+c)^{2} \leqslant 3\left(a^{2}+b^{2}+c^{2}\right) \forall a, b, c>0 .
$$

В этом случае

$$
\begin{aligned}
& \left|U_{6}\right|^{2} \leqslant 3\left(\int_{0}^{\infty} \int_{-\infty}^{\infty}\left|\sum_{\substack{k_{1}+k_{2}=k,\left|k_{1}\right| \leqslant m / 2,\left|k_{2}\right|>m / 2 \\
k_{1}, k_{2} \in \mathbb{Z}_{0}}} v_{k_{1}} v_{k_{2}} e^{i k x} \varphi(x, t)\right| d x d t\right)^{2}+ \\
& +3\left(\int_{0}^{\infty} \int_{-\infty}^{\infty}\left|\sum_{\substack{k_{1}+k_{2}=k,\left|k_{2}\right| \leqslant m / 2,\left|k_{1}\right|>m / 2, k_{1}, k_{2} \in \mathbb{Z}_{0}}} v_{k_{1}} v_{k_{2}} e^{i k x} \varphi(x, t)\right| d x d t\right)^{2}+3\left(\int_{0}^{\infty} \int_{-\infty}^{\infty}\left|\sum_{\substack{k_{1}+k_{2}=k, k_{1}|>m / 2,\\
| k_{2} \mid>m / 2, k_{1}, k_{2} \in \mathbb{Z}_{0}}} v_{k_{1}} v_{k_{2}} e^{i k x} \varphi(x, t)\right| d x d t\right)^{2} .
\end{aligned}
$$

Надо доказать, что $U_{6}$ стремится к нулю при $m \rightarrow \infty$. Имеем 


$$
\begin{aligned}
\left(\int_{0}^{\infty} \int_{-\infty}^{\infty}\left|\sum_{\substack{k_{1}+k_{2}=k,\left|k_{1}\right| \leqslant m / 2,\left|k_{2}\right|>m / 2, k_{1}, k_{2} \in \mathbb{Z}_{0}}}^{\infty} v_{k_{1}} v_{k_{2}} e^{i k x} \varphi(x, t)\right| d x d t\right)^{2} & =\left(\int_{0}^{T} \int_{-R}^{R}\left|\sum_{\substack{k_{1}+k_{2}=k,\left|k_{1}\right| \leqslant m / 2,\left|k_{2}\right|>m / 2, k_{1}, k_{2} \in \mathbb{Z}_{0}}} v_{k_{1}} v_{k_{2}} e^{i k x} \varphi(x, t)\right| d x d t\right)^{2} \leqslant \\
& \leqslant \int_{0}^{T} \int_{-R}^{R}\left|\sum_{\substack{k_{1}+k_{2}=k,\left|k_{1}\right| \leqslant m / 2,\left|k_{2}\right|>m / 2, k_{1}, k_{2} \in \mathbb{Z}_{0}}}^{T} v_{k_{1}, k_{k_{2}}} e^{i k x}\right|^{2} d x d t \int_{-R}^{T}|\varphi(x, t)|^{2} d x d t
\end{aligned}
$$

где

$$
\left|\sum_{\substack{k_{1}+k_{2}=k,\left|k_{1}\right| \leqslant m / 2, k_{2} \mid>m / 2,}} v_{k_{1}} v_{k_{2}} e^{i k x}\right|^{2} \leqslant\left(\sup _{\substack{k_{1} \in \mathbb{Z}_{0},\left|k_{1}\right| \leqslant m / 2}}\left|k_{1}\right|^{\sigma}\left|v_{k_{1}}\right|\right)^{2}\left(\sup _{\substack{k_{2} \in \mathbb{Z}_{0},\left|k_{2}\right|>m / 2}}\left|k_{2}\right|^{\sigma}\left|v_{k_{2}}\right|\right)^{2}\left(\sum_{\substack{\left|k_{1}\right| \leqslant m / 2,\left|k_{2}\right|>m / 2, k_{1}, k_{2} \in \mathbb{Z}_{0}}} \frac{1}{\left|k_{2}\right|^{\sigma}\left|k_{1}\right|^{\sigma}}\right)^{2} .
$$

Отметим, что

$$
\begin{aligned}
& \left(\sup _{\substack{k_{2} \in \mathbb{Z}_{0},\left|k_{2}\right|>m / 2}}\left|k_{2}\right|^{\sigma}\left|v_{k_{2}}\right|\right)^{2}=\sup _{\substack{k_{2} \in \mathbb{Z}_{0} \\
\left|k_{2}\right|>m / 2}}\left|k_{2}\right|^{2 \sigma}\left|v_{k_{2}}\right|^{2} \\
& \left(\sum_{\substack{\left|k_{1}\right| \leqslant m / 2,\left|k_{2}\right|>m / 2,}} \frac{1}{\left|k_{2}\right|^{\sigma}\left|k_{1}\right|^{\sigma}}\right)^{2} \leqslant\left(\sum_{\substack{\left|k_{1}\right| \leqslant m / 2, k_{1} \in \mathbb{Z}_{0}}} \frac{1}{\left|k_{1}\right|^{\sigma}\left|\frac{m}{2}\right|^{\sigma}}\right)^{2} \leqslant \frac{2^{2 \sigma}}{m^{2 \sigma}}\left(\sum_{\substack{\left|k_{1}\right| \leqslant m / 2, k_{1} \in \mathbb{Z}_{0}}} \frac{1}{\left|k_{1}\right|^{\sigma}}\right)^{2} \leqslant \\
& k_{1}, k_{2} \in \mathbb{Z}_{0} \\
& \leqslant\left(\frac{2}{m}\right)^{2 \sigma-1} \sum_{\substack{\left|k_{1}\right| \leqslant m / 2 \\
k_{1} \in \mathbb{Z}_{0}}} \frac{1}{\left|k_{1}\right|^{2 \sigma}} \leqslant\left(\frac{2}{m}\right)^{2 \sigma-1} \sum_{k_{1} \in \mathbb{Z}_{0}} \frac{1}{\left|k_{1}\right|^{2 \sigma}} \leqslant\left(\frac{2}{m}\right)^{2 \sigma-1} c_{2},
\end{aligned}
$$

где

$$
\sum_{k_{1} \in \mathbb{Z}_{0}} \frac{1}{\left|k_{1}\right|^{2 \sigma}}<\infty, \quad \sigma>1 / 2
$$

Вынося супремум и применяя неравенство Гельдера, приходим к оценке

$$
\begin{aligned}
& \left|U_{6}\right|^{2} \leqslant \\
& \leqslant 3\left(\frac{2}{m}\right)^{2 \sigma-1} c_{2} \sup _{t \in \mathbb{R}_{+}} \sup _{\substack{k_{2} \in \mathbb{Z}_{0},\left|k_{2}\right|>m / 2}}\left|k_{2}\right|^{2 \sigma}\left|v_{k_{2}}\right|^{2} \int_{0}^{T} \int_{-R}^{R}|\varphi(x, t)|^{2} d x d t \int_{0}^{T} \int_{-R}^{R} \sup _{\substack{k_{1} \in \mathbb{Z}_{0},\left|k_{1}\right| \leqslant m / 2}}\left|k_{1}\right|^{2 \sigma}\left|v_{k_{1}}\right|^{2} d x d t+ \\
& +3\left(\frac{2}{m}\right)^{2 \sigma-1} c_{2} \sup _{t \in \mathbb{R}_{+}} \sup _{\substack{k_{1} \in \mathbb{Z}_{0},\left|k_{1}\right|>m / 2}}\left|k_{1}\right|^{2 \sigma}\left|v_{k_{1}}\right|^{2} \int_{0}^{T} \int_{-R}^{R}|\varphi(x, t)|^{2} d x d t \int_{0}^{T} \int_{-R}^{R} \sup _{\substack{k_{2} \in \mathbb{Z}_{0},\left|k_{2}\right| \leqslant m / 2}}\left|k_{2}\right|^{2 \sigma}\left|v_{k_{2}}\right|^{2} d x d t+ \\
& +3 \sup _{t \in \mathbb{R}_{+}} \sup _{\substack{k_{2} \in \mathbb{Z}_{0},\left|k_{2}\right|>m / 2}}\left|k_{2}\right|^{2 \sigma}\left|v_{k_{2}}\right|^{2} \int_{0}^{T} \int_{-R}^{R}|\varphi(x, t)|^{2} d x d t \int_{0}^{T} \int_{-R}^{R} \sup _{\substack{k_{1} \in \mathbb{Z}_{0},\left|k_{1}\right|>m / 2}}\left|k_{1}\right|^{2 \sigma}\left|v_{k_{1}}\right|^{2} d x d t\left(\sum_{\substack{k_{1}, k_{2} \in \mathbb{Z}_{0},\left|k_{1}\right|>m / 2,\left|k_{2}\right|>m / 2}} \frac{1}{\left|k_{1}\right|^{\sigma}\left|k_{2}\right|^{\sigma}}\right)^{2},
\end{aligned}
$$


где

$$
\int_{0}^{T} \int_{-R}^{R} \sup _{\substack{k_{1} \in \mathbb{Z}_{0},\left|k_{1}\right| \leqslant m / 2}}\left|k_{1}\right|^{2 \sigma}\left|v_{k_{1}}\right|^{2} d x d t \leqslant c_{1} \int_{0}^{\infty} e^{2 \gamma t} \sup _{\substack{k_{1} \in \mathbb{Z}_{0},\left|k_{1}\right| \leqslant m / 2}}\left|k_{1}\right|^{2 \sigma}\left|v_{k_{1}}\right|^{2} d t=c_{1}\|\widehat{v}\|_{L_{2, \gamma}\left(\mathbb{R}_{+} ; \mathcal{H}_{\sigma}^{(m / 2)}\right)^{2}}^{2}
$$

Остальные интегралы оцениваются таким же образом. Отсюда вытекает, что

$$
\begin{aligned}
&\left|U_{6}\right|^{2} \leqslant\left(\frac{2}{m}\right)^{2 \sigma-1} c_{3} \sup _{t \in \mathbb{R}_{+}} \sup _{\substack{k_{2} \in \mathbb{Z}_{0},\left|k_{2}\right|>m / 2}}\left|k_{2}\right|^{2 \sigma}\left|v_{k_{2}}\right|^{2} \int_{0}^{T} \int_{-R}^{R}|\varphi(x, t)|^{2} d x d t \times\|\widehat{v}\|_{L_{2, \gamma}\left(\mathbb{R}_{+} ; \mathcal{H}_{\sigma}^{(m / 2)}\right)^{+}}^{2} \\
&+\left(\frac{2}{m}\right)^{2 \sigma-1} c_{3} \sup _{t \in \mathbb{R}_{+}} \sup _{\substack{k_{1} \in \mathbb{Z}_{0},\left|k_{1}\right|>m / 2}}\left|k_{1}\right|^{2 \sigma}\left|v_{k_{1}}\right|^{2} \int_{0}^{T} \int_{-R}^{R}|\varphi(x, t)|^{2} d x d t \times\|\widehat{v}\|_{L_{2, \gamma}\left(\mathbb{R}_{+} ; \mathcal{H}_{\sigma}^{(m / 2)}\right)^{+}}^{2} \\
&+c_{2} \sup _{t \in \mathbb{R}_{+}} \sup _{\substack{k_{2} \in \mathbb{Z}_{0},\left|k_{2}\right|>m / 2}}\left|k_{2}\right|^{2 \sigma}\left|v_{k_{2}}\right|^{2} \int_{0}^{T} \int_{-R}^{R}|\varphi(x, t)|^{2} d x d t \times\|\widehat{v}\|_{L_{2, \gamma}\left(\mathbb{R}_{+} ; \mathcal{H}_{\sigma}\right)}^{2} \times \frac{1}{m^{2 \sigma}}\left(\sum_{\substack{k_{1}, k_{2} \in \mathbb{Z}_{0},\left|k_{1}\right|>m / 2,\left|k_{2}\right|>m / 2}} \frac{\left.\left.\left.m^{\sigma}\right|^{\sigma}\left|k_{1}\right|^{\sigma}\right|_{2}\right|^{\sigma}}{2}\right.
\end{aligned}
$$

Лемма 6. Следующая сумма сходится при $\sigma>1$ :

$$
\sum_{\substack{k_{1}, k_{2} \in \mathbb{Z}_{0},\left|k_{1}\right|>m / 2 \\\left|k_{2}\right|>m / 2}} \frac{m^{\sigma}}{\left|k_{1}\right|^{\sigma}\left|k_{2}\right|^{\sigma}}<\infty
$$

Доказательство. Заметим, что сумму можно переписать в виде

$$
S=\sum_{\substack{k_{1}, k_{2} \in \mathbb{Z}_{0},\left|k_{1}\right|>m / 2,\left|k_{2}\right|>m / 2}} \frac{m^{\sigma}}{\left|k_{1}\right|^{\sigma}\left|k_{2}\right|^{\sigma}}=\sum_{\substack{k_{1}, k_{2} \in \mathbb{Z}_{0}, \min \left(\left|k_{1}\right|,\left|k_{2}\right|\right)>m / 2}} \frac{m^{\sigma}}{\left|k_{1}\right|^{\sigma}\left|k_{2}\right|^{\sigma}}
$$

а также имеет место равенство

$$
\left|k_{1}\right|^{\sigma} \times\left|k_{2}\right|^{\sigma}=\left(\max \left(\left|k_{1}\right|,\left|k_{2}\right|\right)\right)^{\sigma}\left(\min \left(\left|k_{1}\right|,\left|k_{2}\right|\right)\right)^{\sigma} .
$$

В этом случае

$$
\begin{aligned}
S= & \sum_{\substack{k_{1}, k_{2} \in \mathbb{Z}_{0}, \min \left(\left|k_{1}\right|,\left|k_{2}\right|\right)>m / 2}} \frac{m^{\sigma}}{\left(\max \left(\left|k_{1}\right|,\left|k_{2}\right|\right)\right)^{\sigma}\left(\min \left(\left|j_{1}\right|,\left|j_{2}\right|\right)\right)^{\sigma}} \leqslant \\
& \leqslant c_{\sigma} \sum_{k_{1}, k_{2} \in \mathbb{Z}_{0}} \frac{1}{\left(\max \left(\left|k_{1}\right|,\left|k_{2}\right|\right)\right)^{\sigma}} \leqslant c_{\sigma} \sum_{k_{1}=1}^{\infty} \frac{1}{\left|k_{1}\right|^{\sigma}}+c_{\sigma} \sum_{k_{2}=1}^{\infty} \frac{1}{\left|k_{2}\right|^{\sigma}} .
\end{aligned}
$$

Поскольку в суммы входят одинаковые слагаемые, имеем

$$
S \leqslant 2 \sum_{k_{1}=1}^{\infty} \frac{1}{\left|k_{1}\right|^{\sigma}}<\infty
$$

если $\sigma>1$. Лемма доказана.

Из ограниченности выражений

$$
\sup _{t \in \mathbb{R}_{+}} \sup _{\substack{k_{1} \in \mathbb{Z}_{0},\left|k_{1}\right|>m / 2}}\left|k_{1}\right|^{2 \sigma}\left|v_{k_{1}}\right|^{2}, \quad \sup _{t \in \mathbb{R}_{+}} \sup _{\substack{k_{2} \in \mathbb{Z}_{0} \\\left|k_{2}\right|>m / 2}}\left|k_{2}\right|^{2 \sigma}\left|v_{k_{2}}\right|^{2}
$$


и норм $\|\widehat{v}\|_{L_{2, \gamma}\left(\mathbb{R}_{+} ; \mathcal{H}_{\sigma}^{(m / 2)}\right)}^{2},\|\widehat{v}\|_{L_{2, \gamma}\left(\mathbb{R}_{+} ; \mathcal{H}_{\sigma}\right)}^{2}$ равномерно по $m$ следует, что

$$
U_{6} \rightarrow 0, \quad m \rightarrow \infty \text {. }
$$

Аналогично доказывается, что

$$
U_{7}=\int_{0}^{\infty} \int_{-\infty}^{\infty}\left(\widehat{u} \widehat{w}-\widehat{u}^{(m)} \widehat{w}^{(m)}\right) \varphi(x, t) d x d t \rightarrow 0, \quad m \rightarrow \infty .
$$

Рассмотрим выражение для нулевой моды

$$
U_{8}=\int_{0}^{\infty} \int_{-\infty}^{\infty}\left(u_{0}-u_{0}^{(m)}\right) \partial_{t} \varphi(x, t) d x d t
$$

Имеет место оценка

$$
\left|I_{8}\right|^{2}=\left(\int_{0}^{\infty} \int_{-\infty}^{\infty}\left|\left(u_{0}-u_{0}^{(m)}\right)\right|\left|\partial_{t} \varphi(x, t)\right| d x d t\right)^{2} .
$$

Применим неравенство Гельдера:

$$
\begin{gathered}
\left|I_{8}\right|^{2} \leqslant \int_{0}^{\infty} \int_{-R}^{R}\left|\left(u_{0}-u_{0}^{(m)}\right)\right|^{2} d x d t \int_{0}^{\infty} \int_{-\infty}^{\infty}\left|\partial_{t} \varphi(x, t)\right|^{2} d x d t \leqslant \\
\leqslant 2 R \lim _{T \rightarrow \infty} \int_{0}^{T} e^{2 \gamma t} e^{-2 \gamma t}\left|\left(u_{0}-u_{0}^{(m)}\right)\right|^{2} d t \int_{0}^{\infty} \int_{-\infty}^{\infty}\left|\partial_{t} \varphi(x, t)\right|^{2} d x d t \leqslant \\
\leqslant 2 R \sup _{t \in \mathbb{R}_{+}} e^{2 \gamma t}\left|\left(u_{0}-u_{0}^{(m)}\right)\right|^{2} \int_{0}^{\infty} e^{-2 \gamma t} d t \int_{0}^{\infty} \int_{-\infty}^{\infty}\left|\partial_{t} \varphi(x, t)\right|^{2} d x d t= \\
=c_{0}\left\|u_{0}-u_{0}^{(m)}\right\|_{L_{\infty, \gamma}\left(\mathbb{R}_{+}\right)}^{2} \int_{0}^{\infty} \int_{-\infty}^{\infty}\left|\partial_{t} \varphi(x, t)\right|^{2} d x d t \rightarrow 0, \quad m \rightarrow \infty
\end{gathered}
$$

Аналогично получаем, что при $m \rightarrow \infty$

$$
\begin{aligned}
& -\int_{0}^{\infty} \int_{-\infty}^{\infty}\left(u_{0}-u_{0}^{(m)}\right) \partial_{x} \varphi(x, t) d x d t- \\
& \quad-\int_{0}^{\infty} \int_{\infty}^{\infty}\left(\frac{1}{\varepsilon} w_{e}^{1 / 2}\left(2 v_{e}^{1 / 2}\left(v_{0}-v_{0}^{(m)}\right)-u_{e}^{1 / 2}\left(w_{0}-w_{0}^{(m)}\right)-w_{e}^{1 / 2}\left(u_{0}-u_{0}^{(m)}\right)\right)\right) \varphi(x, t) d x d t \rightarrow 0 .
\end{aligned}
$$

Запишем оставшиеся выражения через коэффициенты Фурье для $|k| \leqslant m$ :

$$
\begin{gathered}
\int_{0}^{\infty} \int_{-\infty}^{\infty}\left(\frac{d}{d t} u_{0}^{(m)}-\frac{1}{\varepsilon} w_{e}^{1 / 2}\left(2 v_{e}^{1 / 2} v_{0}^{(m)}-u_{e}^{1 / 2} w_{0}^{(m)}-w_{e}^{1 / 2} u_{0}^{(m)}\right)-\right. \\
\left.-\varepsilon w_{e}^{1 / 2} \sum_{\substack{k_{1}+k_{2}=0,\left|k_{1}\right|,\left|k_{k}\right| \leqslant m, k_{1}, k_{2} \in \mathbb{Z}}}\left(v_{k_{1}}^{(m)} v_{k_{2}}^{(m)}-u_{k_{1}}^{(m)} w_{k_{2}}^{(m)}\right)\right) \varphi(x, t) d x d t+
\end{gathered}
$$




$$
\begin{gathered}
+\int_{0}^{\infty} \int_{-\infty}^{\infty} \sum_{\substack{|k| \leqslant m \\
k \in \mathbb{Z}_{0}}}\left(\frac{d}{d t} u_{k}^{(m)}+i k u_{k}^{(m)}-\frac{1}{\varepsilon} w_{e}^{1 / 2}\left(2 v_{e}^{1 / 2} v_{k}^{(m)}-u_{e}^{1 / 2} w_{k}^{(m)}-w_{e}^{1 / 2} u_{k}^{(m)}\right)-\right. \\
\left.-\varepsilon w_{e}^{1 / 2}\left(2 v_{0}^{(m)} v_{k}^{(m)}-u_{0}^{(m)} w_{k}^{(m)}-u_{k}^{(m)} w_{0}^{(m)}\right)\right) e^{i k x} \varphi(x, t) d x d t- \\
-\varepsilon w_{e}^{1 / 2} \int_{0}^{\infty} \sum_{-\infty}^{\infty} \sum_{\substack{k_{1}+k_{2}=k,\left|k_{1}\right| \leqslant m,\left|k_{2}\right| \leqslant m, k_{1}, k_{2} \in \mathbb{Z}_{0}}}^{\infty}\left(v_{k_{1}}^{(m)} v_{k_{2}}^{(m)}-u_{k_{1}}^{(m)} w_{k_{2}}^{(m)}\right) e^{i k x} \varphi(x, t) d x d t
\end{gathered}
$$

В силу построения при $k=0$

$$
\frac{d}{d t} u_{0}^{(m)}-\frac{1}{\varepsilon} w_{e}^{1 / 2}\left(2 v_{e}^{1 / 2} v_{0}^{(m)}-u_{e}^{1 / 2} w_{0}^{(m)}-w_{e}^{1 / 2} u_{0}^{(m)}\right)-\varepsilon w_{e}^{1 / 2} \sum_{\substack{k_{1}+k_{2}=0,\left|k_{1}\right|,\left|k_{2}\right| \leqslant m \\ k_{1}, k_{2} \in \mathbb{Z}}}\left(v_{k_{1}}^{(m)} v_{k_{2}}^{(m)}-u_{k_{1}}^{(m)} w_{k_{2}}^{(m)}\right)=0
$$

а при $|k| \leqslant m-$

$$
\begin{aligned}
& \frac{d}{d t} u_{k}^{(m)}+i k u_{k}^{(m)}-\frac{1}{\varepsilon} w_{e}^{1 / 2}\left(2 v_{e}^{1 / 2} v_{k}^{(m)}-u_{e}^{1 / 2} w_{k}^{(m)}-w_{e}^{1 / 2} u_{k}^{(m)}\right)- \\
&-\varepsilon w_{e}^{1 / 2}\left(2 v_{0}^{(m)} v_{k}^{(m)}-u_{0}^{(m)} w_{k}^{(m)}-u_{k}^{(m)} w_{0}^{(m)}\right)-\varepsilon w_{e}^{1 / 2} \sum_{\substack{k_{1}+k_{2}=k,\left|k_{1}\right| \leqslant m,\left|k_{2}\right| \leqslant m, k_{1}, k_{2} \in \mathbb{Z}_{0}}}\left(v_{k_{1}}^{(m)} v_{k_{2}}^{(m)}-u_{k_{1}}^{(m)} w_{k_{2}}^{(m)}\right)
\end{aligned}
$$

Таким образом, предельные функции $(\widehat{u}, \widehat{v}, \widehat{w})$ есть слабое решение задачи Коши $(3)$.

Теорема 8. Пусть $\sigma>2$ и выполнено условие $(24)$. Тогда $(\widehat{u}, \widehat{v}, \widehat{w}) \in W_{2}^{1}\left(\mathbb{R}_{+} \times R^{1}\right)$ является классическим решением для почти всех $(x, t) \in \mathbb{R}_{+} \times R^{1}$.

\section{СПИСОК ЛИТЕРАТУРЫ}

1. Василъева О. А. Численное исследование системы уравнений Годунова-Султангазина. Периодический случай// Вестн. МГСУ. - 2016. - № 4. - С. 27-35.

2. Васильева О. А., Духновский C. А. Условие секулярности кинетической системы Карлемана// Вестн. МГСУ. - 2015. - № 7. - С. 33-40.

3. Веденяпин В. В. Кинетические уравнения Больцмана и Власова. - М.: Физматлит, 2001.

4. Годунов C. K., Султангазин У. М. О дискретных моделях кинетического уравнения Больцмана// Усп. мат. наук. - 1971. - 26, № 3. - С. 3-51.

5. Духновский C. A. О скорости стабилизации решений задачи Коши для уравнения Карлемана с периодическими начальными данными// Вестн. Самар. гос. техн. ун-та. Сер. физ.-мат. науки. - 2017. 21, № 1. - С. 7-41.

6. Ильин О. В. Изучение существования решений и устойчивости кинетической системы Карлемана// Ж. вычисл. мат. мат. физ. - 2007. - 47, № 12. - С. 2076-2087.

7. Радкевич Е. В. О дискретных кинетических уравнениях// Докл. РАН. - 2012. - 447, № 4. - С. 369373.

8. Радкевич E. В. О поведении на больших временах решений задачи Коши для двумерного кинетического уравнения// Совр. мат. Фундам. напр. - 2013. - 47. - C. 108-139.

9. Buslaev V. S., Komech A., Kopylova E. A., Stuart D. On asymptotic stability of solitary waves in nonlinear Schrödinger equation// Commun. Partial Differ. Equ. — 2008. — 33, № 4. — P. 669-705.

10. Kopylova E. A. On long-time decay for magnetic Schrödinger and Klein-Gordon equations// Tp. Mat. ин-та им. В. А. Стеклова. - 2012. - 278. - С. 129-137.

11. Radkevich E. V., Vasil'eva O. A., Dukhnovskii S. A. Local equilibrium of the Carleman equation// J. Math. Sci. - 2015. - 207, № 2. - P. 296-323. 
12. Vasil'eva O. A., Dukhnovskii S. A., Radkevich E. V. On the nature of local equilibrium in the Carleman and Godunov-Sultangazin equations// J. Math. Sci. — 2018. — 235, № 4. - P. 393-453.

Духновский Сергей Анатольевич

Национальный исследовательский

Московский государственный строительный университет, Москва

E-mail: sergeidukhnvskijj@rambler.ru 